\title{
Option Pricing in a One-Dimensional Affine Term Structure Model via Spectral Representations
}

DOI:

10.1137/16M1098267

\section{Document Version}

Final published version

Link to publication record in Manchester Research Explorer

\section{Citation for published version (APA):}

Chazal, M., Loeffen, R., \& Patie, P. (2018). Option Pricing in a One-Dimensional Affine Term Structure Model via Spectral Representations. SIAM Journal on Financial Mathematics, 9(2), 634-664.

https://doi.org/10.1137/16M1098267

\section{Published in:}

SIAM Journal on Financial Mathematics

\section{Citing this paper}

Please note that where the full-text provided on Manchester Research Explorer is the Author Accepted Manuscript or Proof version this may differ from the final Published version. If citing, it is advised that you check and use the publisher's definitive version.

\section{General rights}

Copyright and moral rights for the publications made accessible in the Research Explorer are retained by the authors and/or other copyright owners and it is a condition of accessing publications that users recognise and abide by the legal requirements associated with these rights.

\section{Takedown policy}

If you believe that this document breaches copyright please refer to the University of Manchester's Takedown Procedures [http://man.ac.uk/04Y6Bo] or contact uml.scholarlycommunications@manchester.ac.uk providing relevant details, so we can investigate your claim.

\section{OPEN ACCESS}




\title{
Option Pricing in a One-Dimensional Affine Term Structure Model via Spectral Representations*
}

\author{
M. Chazal ${ }^{\dagger}$, R. Loeffen ${ }^{\ddagger}$, and P. Patie ${ }^{\dagger}$
}

\begin{abstract}
Under a mild condition on the branching mechanism, we provide an eigenvalue expansion for the pricing semigroup in a one-dimensional positive affine term structure model. This representation, which is based on results from Ogura [Publ. Res. Inst. Math. Sci., 6 (1970), pp. 307-321], recently improved by the authors in [J. Math. Anal. Appl., 459 (2018), pp. 619-660], allows us to get analytical expressions for the prices of interest rate sensitive European claims. As the pricing semigroups are non-self-adjoint linear operators, the computation of eigenfunctions and co-eigenmeasures is required in the expansions. We describe comprehensive methodologies to characterize these spectral objects from merely the knowledge of the branching and immigration mechanisms. To illustrate the computation power and advantages of our approach, we develop comparison analysis with FourierLaplace inversion techniques for some examples. Numerical experiments are provided and show that the spectral approach allows one to quickly price European vanilla options on bonds and yields for a whole range of strikes and maturities.
\end{abstract}

Key words. option pricing, affine terms structure model, eigenvalue expansions, non-self-adjoint, numerical methods

AMS subject classifications. Primary, 00A30; Secondary, 00A22, 03E20

DOI. $10.1137 / 16 \mathrm{M} 1098267$

1. Introduction. Kawazu and Watanabe [19] introduced the class of one-dimensional continuous-state branching processes with immigration (for short, CBI-processes), which appear in a natural way as limits of Galton-Watson branching processes with immigration. In mathematical finance CBI-processes are also known as one-dimensional, positive, timehomogeneous affine processes. Their affine properties offer some interesting features for modeling the dynamics of financial assets such as the instantaneous interest rates and the stochastic volatility of asset prices, and also in credit risk. This is one of the reasons why they have received the attention of many researchers over the last decade and there exists a huge amount of papers devoted to the study of their fine properties or applications in mathematical finance; see, e.g., Duffie, Pan, and Singleton [10], Filipović [11], Duffie, Filipović, and Schachermayer [8], Kallsen [17], Patie [30], and Li [25], to name but a few.

Although these models have very attractive practical characteristics, it is usually difficult, beside the diffusion case, i.e., the Cox-Ingersoll-Ross (CIR) model, to obtain closed-form expressions for the prices of interest rate derivatives. The first aim of this paper is to fill in

\footnotetext{
${ }^{*}$ Received by the editors October 11, 2016; accepted for publication (in revised form) March 26, 2018; published electronically May 24, 2018.

http://www.siam.org/journals/sifin/9-2/M109826.html

${ }^{\dagger}$ School of Operations Research and Information Engineering, Cornell University, Ithaca, NY 14853 (mc969@ cornell.edu, pp396@cornell.edu).

${ }^{\ddagger}$ School of Mathematics, University of Manchester, Manchester M13 9PL, UK (ronnie.loeffen@manchester.ac.uk).
} 
this gap by providing, under a technical condition on the branching mechanism, an eigenvalue expansion for the pricing semigroup when acting on some linear space that we characterize. This spectral representation, which is based on original studies initiated by Ogura in [28] and [29] and further developed by the authors in [4], yields to an explicit representation of vanilla type options in the one-dimensional positive affine term structure model. We mention that Davydov and Linetsky [7], Gorovoi and Linetsky [14], Linetsky [26], and Boyarchenko and Levendorskii [2] suggested several applications of spectral expansions for the pricing of securities in the framework of linear diffusions based models. This decomposition of prices relies upon the seminal work of McKean [27] on spectral theory of one-dimensional diffusions which we recall generates self-adjoint linear operators. We emphasize that, beyond the diffusion case, the semigroups associated to one-dimensional Markov processes are in general non-selfadjoint, even non-normal, and the spectral theory of such linear operators is fragmentally understood. We refer, for instance, to Davies [5], Patie and Savov [31], [32], Patie and Zhao [33], and the references therein, for recent accounts and developments in this area.

Another advantage of affine processes in mathematical finance is that after solving the so-called generalized Riccati equations, one obtains the Laplace transform of the transition distribution and this opens the door to fast pricing of European-type options via one Laplace/Fourier inversion; see, e.g., Duffie, Pan, and Singleton [10]. Moreover, in a Markovian short rate model in which the short rate process is modeled by a CBI-process, the zero-coupon bond prices are exponentially affine in the short rate and this leads to fast pricing of vanilla options on bonds and yields. The second aim of this paper is to offer an alternative numerical approach to approximate options prices by investigating the numerical performance of the eigenvalues expansion for vanilla type options compared to the approximation techniques based on Fourier-Laplace inversion methods. This new perspective leads to an extremely fast method for computing option prices, as well as risk-sensitive quantities, if all the components, that is, the eigenfunctions and co-eigenmeasures, of the spectral representation are known explicitly. However, one quickly realizes that explicit expressions of these objects (which are characterized via either their generating function or their Laplace transform) cannot be found in most cases. Therefore, on the one hand, we present an original algorithm to compute the coefficients of the Sheffer polynomials arising in the expression of the eigenfunctions. Moreover, we introduce an option pricing method based upon the spectral representation of Ogura [29], but where the terms involving the co-eigenmeasures are computed by Laplace inversion. This latter method still provides a significant speed advantage when one computes option prices for multiple expiry times. We point out that although in this paper we focus on the application of the spectral expansion approach to option pricing in an affine short rate model, one can naturally use the method as well in other areas of mathematical finance that involve one-dimensional, positive, affine processes, like, for instance, the affine LIBOR model of Keller-Ressel, Papapantoleon, and Teichmann [21].

The contributions of the paper, in particular in comparison to our paper [4] and Ogura's papers [28], [29], are the following. First, we work out in detail the spectral representation in [4] for the application of option pricing in a one-dimensional affine short rate model. Second, since the spectral representation is a series, one needs to truncate the infinite sum for numerical computation of the option prices. We provide a result on the speed of convergence as the number of terms grows to infinity. Third, we describe how one can compute the components of the spectral representation. In particular we provide a new way of computing the eigenfunctions.

Copyright (c) by SIAM. Unauthorized reproduction of this article is prohibited. 
We further compare the two spectral methods with the method of Laplace inversion using the generalized Riccati equations. Finally, we conduct some numerical experiments to illustrate how well the two spectral methods perform in practice.

The remainder of this paper is organized as follows. In the next section, after reviewing the main concepts underlying the one-dimensional affine pricing semigroup, we present its eigenvalues expansion together with some illustrative examples. In section 3, we describe three methods for pricing European bond and yield options in an affine term structure model. Two of the three methods are based upon the spectral representation and we discuss their benefits and drawbacks. In section 4 we detail two examples, whereas in section 5 we provide some numerical results that indicate the performance of the two spectral methods. Section 6 concludes the paper. All the proofs are put in the appendix.

2. Spectral expansion of the pricing semigroup in an affine term structure model. In this part, we present the spectral expansion of the pricing semigroup of a one-dimensional affine term structure model on some linear space to be specified. This yields closed-form expressions for the valuation of a large class of European options. To highlight our approach, we shall describe in detail the case of the call option on a zero-coupon bond and the put option on the yield. In addition to its spectral expansion, we obtain series expansions for the crossderivatives of any order of the pricing semigroup, namely, of the function $(t, x) \longmapsto P_{t} f(x)$. This provides explicit expressions of risk sensitivities without any additional computational effort in comparison to the spectral expansion of the pricing semigroup itself. From now on, we assume that the risk-neutral dynamics of the short rate process $r=\left(r_{t}\right)_{t \geq 0}$ taking values in $\mathbb{R}_{>0}^{+}=[0, \infty)$ and defined on the probability space $\left(\Omega, \mathcal{F},\left(\mathcal{F}_{t}\right)_{t \geq 0},\left(\mathbb{P}_{x}\right)_{x \geq 0}\right)$, is a conservative $\mathrm{CB} I$-process. Next, let $P=\left(P_{t}\right)_{t \geq 0}$ be the family of linear operators, defined, for any $f$ in $\mathcal{B}\left(\mathbb{R}_{\geq 0}^{+}\right)$, the set of bounded borelian functions on $\mathbb{R}_{\geq 0}^{+}$and any $t, x \geq 0$, by

$$
P_{t} f(x)=\mathbb{E}_{x}\left[e^{-\int_{0}^{t} r_{s} d s} f\left(r_{t}\right)\right] .
$$

The operator $P_{t}$ is usually called the pricing semigroup as the right-hand side corresponds to the current price of an European option on the short rate with payoff $f\left(r_{t}\right)$ at maturity $t$. Moreover since $r$ is a Feller process, from the general theory of Markov processes, we get that $P$ is the semigroup of a Feller process obtained as the semigroup associated to $r$ subordinated by the discount factor multiplicative functional $\left(e^{-\int_{0}^{t} r_{s} d s}\right)_{t>0}$; see [1]. A nice feature of the class of CBI-semigroups is that it is invariant by this type of subordination. In other words, $P$ is also the semigroup of a CBI-process. Following Kawazu and Watanabe's description of CBI-processes in [19], Filipović [11] showed that the transition kernel associated with the pricing semigroup $P$ is characterized in terms of its Laplace transform as follows. Writing $e_{\lambda}(x)=e^{-\lambda x}$, one has, for any $t, x, \lambda \geq 0$,

$$
P_{t} e_{\lambda}(x)=e^{-\Phi_{t}(\lambda)-\Psi_{t}(\lambda) x}
$$

where the CBI exponents $\Psi_{t}$ and $\Phi_{t}$ solve, for any fixed $\lambda \geq 0$, the so-called generalized Riccati equations,

$$
\begin{aligned}
\frac{\partial}{\partial t} \Psi_{t}(\lambda) & =-\psi\left(\Psi_{t}(\lambda)\right), \quad \Psi_{0}(\lambda)=\lambda \\
\Phi_{t}(\lambda) & =\int_{0}^{t} \phi\left(\Psi_{s}(\lambda)\right) d s
\end{aligned}
$$

Copyright (c) by SIAM. Unauthorized reproduction of this article is prohibited. 
with the branching and immigration mechanisms given respectively by

$$
\begin{aligned}
& \psi(u)=\sigma^{2} u^{2}+\beta u-1-\int_{0}^{\infty}\left(1-e^{-u y}-u(1 \wedge y)\right) \Pi(d y), \\
& \phi(u)=b u+\int_{0}^{\infty}\left(1-e^{-u y}\right) \mu(d y)
\end{aligned}
$$

where $\sigma, b \geq 0, \beta \in \mathbb{R}$ and $\Pi$ and $\mu$ are nonnegative Borel measures on $(0, \infty)$ satisfying

$$
\int_{0}^{\infty}\left(1 \wedge y^{2}\right) \Pi(d y)+\int_{0}^{\infty}(1 \wedge y) \mu(d y)<\infty
$$

Endowing $C_{0}\left(\mathbb{R}_{>0}^{+}\right)$, the space of continuous functions on $\mathbb{R}_{\geq 0}^{+}$vanishing at $\infty$, with the uniform topology, it is well-known that the pricing semigroup $P$ is a Feller semigroup and its infinitesimal generator $\mathcal{A}$ is the linear operator defined, for any $f \in \mathcal{D} \subset C_{0}\left(\mathbb{R}_{\geq 0}^{+}\right)$, the domain of $\mathcal{A}$, by $\mathcal{A} f(x)=\lim _{t \downarrow 0} \frac{P_{t} f(x)-f(x)}{t} \in C_{0}\left(\mathbb{R}_{\geq 0}^{+}\right)$. Further, for at least functions $f$ in the linear hull of $\left\{e_{\lambda}: \lambda>0\right\}$, we have

$$
\begin{aligned}
\mathcal{A} f(x)= & \sigma^{2} x f^{\prime \prime}(x)+(b-\beta x) f^{\prime}(x)-x f(x)+x \int_{0}^{\infty}\left(f(x+y)-f(x)-f^{\prime}(x)(1 \wedge y)\right) \Pi(d y) \\
& +\int_{0}^{\infty}(f(x+y)-f(x)) \mu(d y)
\end{aligned}
$$

see, e.g., [19]. For the representation of a CBI-process as the solution of a stochastic differential equation we refer to [6]. Observe that $r$ is conservative (i.e., $\mathbb{P}_{x}\left(r_{t}=\infty\right)=0$ for all $x, t \geq 0$ ) if and only if for all $\epsilon>0$,

$$
\int_{0}^{\epsilon} \frac{d u}{|\psi(u)+1|}=\infty
$$

(see, e.g., [11, Theorem 4.11]) and that this condition is satisfied in the case $\int_{1}^{\infty} y \Pi(d y)<\infty$, since this implies $\left|\psi^{\prime}\left(0^{+}\right)\right|<\infty$. Next, since an $S$-bond, that is, a zero-coupon bond maturing at $S$, gives a payment of 1 at time $S$, we get that its price at time $T \leq S$, denoted by $B_{T}(S)$, is given, with $\tau=S-T$, by

$$
B_{T}(S)=\mathbb{E}\left[\mathrm{e}^{-\int_{T}^{S} r_{s} \mathrm{~d} s} \mid \mathcal{F}_{T}\right]=\mathbb{E}_{r_{T}}\left[\mathrm{e}^{-\int_{0}^{\tau} r_{s} \mathrm{~d} s}\right]=e^{-\Phi(\tau)-\Psi(\tau) r_{T}},
$$

where the last identity follows from (2.2), and, for the sake of simplicity, we have set, for any $t \geq 0, \Phi(t)=\Phi_{t}(0)$ and $\Psi(t)=\Psi_{t}(0)$. Thus, from (2.9), we see that the price of a zerocoupon bond depends in an exponential-affine way on the short rate. In the literature this is referred to as the short rate model providing an affine term structure. Filipović [11] showed that essentially a CBI-process is the only model on $\mathbb{R}_{\geq 0}^{+}$for the short rate that provides an affine term structure. 
2.1. Spectral decomposition of the pricing semigroup. Before stating the eigenvalue expansions of the pricing semigroup, we start by introducing some notation and some preliminary results. Throughout, we assume the following condition on the branching mechanism $\psi$ defined in (2.5):

$$
\int_{\lambda}^{\infty} \frac{d u}{|\psi(u)|}<\infty \quad \text { for large } \lambda
$$

Remark 2.1. It is worth pointing out that following the lines of [4, Lemma 2.1] and its proof, one can show that this condition, which is the only requirement on the mechanisms to ensure the spectral reduction of the pricing semigroup, can be expressed in terms of the Lévy characteristics in (2.5). Indeed, (2.10) holds if and only if

$$
\sigma>0 \text { or } \int_{0}^{\lambda} \frac{d v}{\int_{0}^{v} \int_{r}^{1} \Pi(y, \infty) d y d r}<\infty \text { for some } \lambda>0 .
$$

Note that this latter integral test holds if the tail of the Lévy measure $\Pi(y, \infty)$ is regularly varying at zero of index $-2<\alpha<-1$. However, it fails if $\int_{0}^{1} \Pi(y, \infty) d y<\infty$, that is, when the underlying Lévy process has paths of bounded variations, a property that is shared with the short rate process $r$.

Next, since $\psi$ is convex and continuous on $\mathbb{R}_{\geq 0}^{+}$with $\psi(0)=-1$ and it is not difficult to check, under assumption (2.10), that $\psi(u)>0$ for large $u$, we have that there exists a unique positive root of the equation $\psi(u)=0$ which we denote by $\theta$. Note that $\psi$ is positive and increasing on $(\theta, \infty)$ and $\psi^{\prime}(\theta)>0$. In what follows, for a function $f$ that is well-defined at 0 , we shall denote by $R_{f} \geq 0$ the radius of convergence of its Taylor series at 0 . Clearly,

$$
R_{\psi}=\inf \left\{u \geq 0: \int_{1}^{\infty} e^{u y} \Pi(d y)=\infty\right\}, \quad R_{\phi}=\inf \left\{u \geq 0: \int_{1}^{\infty} e^{u y} \mu(d y)=\infty\right\} .
$$

As $\psi$ is convex, there might be a second root of $\psi$ and we let $\underline{\theta}:=\inf \left\{u \in\left(0, R_{\psi}\right): \psi(-u)=0\right\}$ with the understanding that the infimum of an empty set is equal to $+\infty$.

Now we introduce the following two functions, derived from the CBI-semigroup mechanisms, that are key elements for the spectral representation of the pricing semigroup. We set

$$
\begin{aligned}
& A(\lambda)=\exp \left(-\psi^{\prime}(\theta) \int_{\lambda}^{\infty} \frac{d u}{\psi(u)}\right), \quad \lambda \geq \theta, \\
& F(\lambda)=\int_{\theta}^{\lambda} \frac{\phi(u)-\phi(\theta)}{\psi(u)} d u, \quad \lambda \geq \theta .
\end{aligned}
$$

Note that by assumption (2.10) we have

$$
\psi(\theta)=0, \psi^{\prime}(\theta)>0, \int_{\lambda}^{\infty} \frac{d u}{\psi(u)}<\infty \text { for all } \lambda>\theta \text { and } \int_{\theta}^{\infty} \frac{d u}{\psi(u)}=\infty
$$


and we refer the reader to [25, Theorem 3.8] for a proof of the last statement. From Lemma A.1 below we know that $A$ and $F$ can be extended to a larger domain including the point 0 and in particular $R_{A}=R_{\psi} \wedge \underline{\theta}$ and $R_{F}=R_{\psi} \wedge R_{\phi} \wedge \underline{\theta}$. Note that for $\lambda \in\left(-R_{A}, \theta\right)$ the right-hand side of (2.11) does not make sense. Instead we can use the alternative representation,

$$
A(\lambda)=\frac{\int_{\theta}^{\lambda} \exp \left(-\int_{\theta}^{v} \frac{\psi^{\prime}(u)-\psi^{\prime}(\theta)}{\psi(u)} d u\right) d v}{\int_{\theta}^{\infty} \exp \left(-\int_{\theta}^{v} \frac{\psi^{\prime}(u)-\psi^{\prime}(\theta)}{\psi(u)} d u\right) d v}, \quad \lambda \in\left(-R_{A}, \infty\right) \cup\{0\} ;
$$

see Lemma A.1. Hence $A$ is an increasing function on $\left(-R_{A}, \infty\right)$ with $A(\theta)=0$ and $A(\infty)=1$. Denoting by $\bar{A}$ the inverse of $A$, we have that $\bar{A}:\left(A\left(-R_{A}\right), 1\right) \rightarrow\left(-R_{A}, \infty\right)$ is increasing. Moreover, $A$ is analytic at $\theta$ with $A^{\prime}(\theta)>0$; see Lemma A.1. Consequently, invoking the Lagrange inversion theorem, one gets that $\bar{A}$ is analytic at zero, i.e., $R_{\bar{A}}>0$. By Lemma A.2 below the quantities $\Psi_{t}(\lambda)$ and $\Phi_{t}(\lambda)$ appearing in (2.2) can, for all $t \geq 0$, be extended to a larger domain and be expressed as

$$
\begin{aligned}
\Psi_{t}(\lambda) & =\bar{A}\left(e^{-\psi^{\prime}(\theta) t} A(\lambda)\right), \quad \lambda \in\left(-R_{A}, \infty\right) \cup\{0\} \quad \text { and } \quad t \geq 0, \\
e^{-\Phi_{t}(\lambda)} & =e^{-\phi(\theta) t} e^{F\left(\Psi_{t}(\lambda)\right)-F(\lambda)}, \quad \lambda \in\left(-R_{F}, \infty\right) \cup\{0\} \quad \text { and } \quad t \geq 0,
\end{aligned}
$$

which provides an alternative representation, alternative to (2.3) and (2.4), of the CBI exponents.

Since $F$ is analytic at $\theta$ (see Lemma A.1), it follows that, for any $x \geq 0$, the mapping

$$
z \longmapsto G_{x}(z)=e^{F(\bar{A}(z))-x(\bar{A}(z)-\theta)}
$$

is analytic at 0 . Recalling that $\bar{A}(0)=\theta$, we may thus let $\left(\mathcal{L}_{n}\right)_{n \geq 0}$ be the family of Sheffer polynomials whose generating function is $G_{x}(z)$, i.e., for any $x \geq 0$,

$$
\sum_{n=0}^{\infty} \mathcal{L}_{n}(x) z^{n}=G_{x}(z), \quad|z|<R_{G}
$$

where $R_{G}$ is the common radius of convergence of the Taylor series of $G_{x}$ for any $x>0$, meaning that $R_{G}$ does not depend on $x>0$. Clearly, the radius of convergence of the Taylor series of $G_{0}$ is not smaller than $R_{G}$. We set

$$
\underline{\mathrm{T}}=-\frac{\ln \left(R_{G}\right)}{\psi^{\prime}(\theta)}
$$

Note that as $A(\infty)=1$, we must have $R_{\bar{A}} \leq 1$ and thus $R_{G} \leq 1$ and $\underline{\mathrm{T}} \geq 0$.

In order to describe the set of payoff functions for which we are able to obtain a series expansion of the associated option price, we first set, for $T>\underline{\mathrm{T}}$,

$$
\bar{\lambda}_{T}=\min \left(-\bar{A}_{T} \mathbb{I}_{\{\underline{\mathrm{T}}<T<\mathcal{T}+\underline{\mathrm{T}}\}}+R_{A} \mathbb{I}_{\{T \geq \mathcal{T}+\underline{\mathrm{T}}\}}, R_{\phi}\right),
$$

where $\bar{A}_{T}=\bar{A}\left(2-e^{\psi^{\prime}(\theta)(T-\underline{T})}\right)$ and $\mathcal{T}=\frac{1}{\psi^{\prime}(\theta)} \ln \left(2-A\left(-R_{A}\right)\right)$, where $A\left(-R_{A}\right):=\lim _{\lambda \downarrow-R_{A}}$ $A(\lambda) \in[-\infty, \infty)$. It can be checked in a straightforward way that $\bar{\lambda}_{T} \leq R_{F}$. Next let $\mathcal{P}_{\lambda}$, with $\lambda \in \mathbb{R}$, be the payoff space defined by 


$$
\mathcal{P}_{\lambda}=\left\{f: \mathbb{R}_{\geq 0}^{+} \rightarrow \mathbb{R} \text { measurable }: f=O\left(e^{-\lambda x}\right) \text { a.e. }\right\},
$$

that is, $f \in \mathcal{P}_{\lambda}$ if there exists $C>0$ such that $|f(x)| \leq C e^{-\lambda x}$ for almost every $x \geq 0$.

We continue with describing the components of the spectral representation. By Lemma A.1, we can define $\nu$, respectively, $\omega$, as a nonnegative integrable function on $\mathbb{R}^{+}:=(0, \infty)$ whose Laplace transform takes the form

$$
\int_{0}^{\infty} e^{-\lambda y} \nu(y) d y=e^{-F(\lambda)}-e^{-F(\infty)}, \quad \lambda \in\left(-R_{F}, \infty\right) \cup\{0\},
$$

respectively,

$$
\int_{0}^{\infty} e^{-\lambda y} \omega(y) d y=1-A(\lambda), \quad \lambda \in\left(-R_{A}, \infty\right) \cup\{0\}
$$

We further set for $n \geq 1$,

$$
\mathcal{W}_{n}(y)=\sum_{j=1}^{n}\left(\begin{array}{l}
n \\
j
\end{array}\right)(-1)^{j} \omega^{* j}(y)
$$

where $\omega^{* 1}=\omega$, and, for any $n \geq 2$,

$$
\omega^{* n}(y)=\omega^{* n-1} * \omega(y),
$$

where $*$ stands for the standard convolution, i.e., $f * g(y)=\int_{0}^{y} f(y-x) g(x) d x$. Next, let $\nu_{0}=\nu$ and for $n \geq 1$,

$$
\nu_{n}(y)=e^{-F(\infty)} \mathcal{W}_{n}(y)+\mathcal{W}_{n} * \nu(y)+\nu(y), \quad y>0 .
$$

Finally, for $n \geq 0$, we write

$$
\lambda_{n}=\phi(\theta)+n \psi^{\prime}(\theta), \quad \mathcal{L}_{n}^{\theta}(x)=e^{-\theta x} \mathcal{L}_{n}(x),
$$

where $\mathcal{L}_{n}$ is defined in (2.18), and for any function $f$, we write

$$
\mathcal{V}_{n} f=\int_{0}^{\infty} f(y) \mathcal{V}_{n}(d y)=\int_{0}^{\infty} f(y) \nu_{n}(y) d y+f(0) e^{-F(\infty)},
$$

where $\mathcal{V}_{n}(d y)=\nu_{n}(y) d y+e^{-F(\infty)} \delta_{0}(d y)$ with $\delta_{0}$ denoting the Dirac measure at 0 .

We now have all the ingredients to state the spectral representation theorem for the pricing semigroup of the one-dimensional positive affine term structures whose branching mechanism satisfy the condition (2.10). Parts (1) and (2) of the theorem contain the series representation itself along with some smoothness properties and are based on the results in [4]. We refer the reader to [4] for more details and other important developments about the smoothness of the CBI transition kernels. Furthermore, part (3) of the theorem gives a result on the speed of convergence of the series, which is completely new.

Theorem 2.2. Let $T>\underline{\mathrm{T}}$ and $f \in \mathcal{P}_{\lambda}$ for some $\lambda>-\bar{\lambda}_{T}$, where we recall that $\bar{\lambda}_{T}$ is defined in $(2.20)$. 
(1) The price at time 0 of the European option with payoff $f\left(r_{T}\right)$ at maturity time $T$ is given, for any $x \geq 0$, by

$$
P_{T} f(x)=\sum_{n=0}^{\infty} e^{-\lambda_{n} T} \mathcal{L}_{n}^{\theta}(x) \mathcal{V}_{n} f
$$

where the series, for any $T>\underline{\mathrm{T}}$, is locally uniformly convergent in $x$.

(2) The mapping $(T, x) \longmapsto P_{T} f(x) \in C^{\infty^{2}}\left((\underline{\mathrm{T}}, \infty) \times \mathbb{R}^{+}\right)$with, for all integers $p, k \geq 0$,

$$
\frac{\partial^{p}}{\partial T^{p}} \frac{\partial^{k}}{\partial x^{k}} P_{T} f(x)=\sum_{n=0}^{\infty}\left(-\lambda_{n}\right)^{p} e^{-\lambda_{n} T} \frac{\partial^{k}}{\partial x^{k}} \mathcal{L}_{n}^{\theta}(x) \mathcal{V}_{n} f
$$

where the series is locally uniformly convergent in $x$.

(3) Let $S=S(T, \lambda)=\psi^{\prime}(\theta)(T-\underline{\mathrm{T}})-\ln (2-A(\lambda))$. Then $S>0$ and for any $\varepsilon \in(0, S)$ there exists $M=M(\varepsilon)>0$ such that, for any integer $k \geq 0$, there exist $C=C(k, \varepsilon, \lambda, f)>0$ such that for all $x \geq 0$ and $N \geq 1$,

$$
\left|\frac{\partial^{k}}{\partial x^{k}} P_{T} f(x)-\sum_{n=0}^{N-1} e^{-\lambda_{n} T} \frac{\partial^{k}}{\partial x^{k}} \mathcal{L}_{n}^{\theta}(x) \mathcal{V}_{n} f\right| \leq C e^{(M-\theta) x-\phi(\theta) T} \frac{e^{-(S-\varepsilon) N}}{1-e^{-(S-\varepsilon)}} .
$$

Let us stress that the spectral ingredients in the expansion (2.28) are directly characterized in terms of the pricing semigroup mechanisms, or equivalently of the short rate mechanisms. The spectral expansion approach dispenses with solving the Riccati equations satisfied by the CBI exponents. It determines the pricing semigroup without resorting to the explicit knowledge and the analytical inversion of its Laplace transform. We further mention that the expression (2.29) reveals that the spectral method allows one to compute easily classical sensitivities with respect to the variables $x$ and $T$, which are useful for interest rate risk management as explained in Jarrow and Turnbull [16].

The next theorem is an improvement upon Theorem 2.2 for the special case where $f=e_{\lambda}$. Recall from (2.9) that the case $f=e_{0}$ is important for determining bond prices. Thus, next to (2.2) in combination with (2.3)-(2.4) and (2.2) in combination with (2.15)-(2.16), this theorem gives a third representation for the Laplace transform of the short rate process (provided the condition (2.30) below is met). Compared to the other two, this representation has the advantage that it is more explicit since one does not need to determine the CBI exponents $\Psi_{T}$ and $\Phi_{T}$ or the inverse function $\bar{A}$ and further it is the most convenient one when one wants to compute derivatives with respect to $T$. Obtaining bond prices quickly is in particular important for calibrating a given model for the short rate to a term structure of interest rates. Although the spectral representation might well be faster than solving numerically the generalized Riccati equations, the speed advantage will not be that great since for bond prices the Riccati equations (2.3)-(2.4) need to be solved only once, namely, for $\lambda=0$. The real speed advantage of the spectral representation comes with the pricing of interest rate derivatives rather than with the calibration of the term structure, as will become clear by the end of the paper.

Theorem 2.3. Let $T \geq 0$ and $\lambda \in\left(-R_{F}, \infty\right) \cup\{0\}$ be such that 


$$
|A(\lambda)|<e^{\psi^{\prime}(\theta)(T-\underline{\mathrm{T}})} .
$$

Then $(2.28)$ and (2.29) hold for $f=e_{\lambda}$. Further, let $\widetilde{S}=\widetilde{S}(T, \lambda)=\psi^{\prime}(\theta)(T-\underline{\mathrm{T}})-\ln (|A(\lambda)|)$. Then $\widetilde{S}>0$ and for any $\varepsilon \in(0, \widetilde{S})$ there exists $M=M(\varepsilon)>0$ such that, for any integer $k \geq 0$, there exists $C=C(k, \varepsilon, \lambda)>0$ such that for all $x \geq 0$ and $N \geq 1$,

$$
\left|\frac{\partial^{k}}{\partial x^{k}} P_{T} e_{\lambda}(x)-\sum_{n=0}^{N-1} e^{-\lambda_{n} T} \frac{\partial^{k}}{\partial x^{k}} \mathcal{L}_{n}^{\theta}(x) \mathcal{V}_{n} e_{\lambda}\right| \leq C e^{(M-\theta) x-\phi(\theta) T} \frac{e^{-(\widetilde{S}-\varepsilon) N}}{1-e^{-(\widetilde{S}-\varepsilon)}}
$$

Remark 2.4. Since $A$ is a strictly increasing function with $A(\theta)=0$ and $A(\infty)=1$, it follows that (2.30) is in particular satisfied when $\lambda \geq \theta$ and $T \geq \underline{\mathrm{T}}$. Further for $T>\mathrm{T}$, the requirement $\lambda>-\bar{\lambda}_{T}$ in Theorem 2.2 is equivalent to $\lambda>-R_{F}$ and $2-A(\lambda)<e^{\psi^{\prime}(\theta)(T-\underline{T})}$. Because of the aforementioned properties, $2-A(\lambda)>\max (|A(\lambda)|, 1)$, which implies that Theorem 2.3 is indeed an improvement over Theorem 2.2 for the case $f=e_{\lambda}$.

The representation (2.28) can be seen as a spectral type representation of the pricing semigroup $P$. Indeed, the sequence of functions $\left(\mathcal{L}_{n}^{\theta}\right)_{n \geq 0}$ turns out to be a sequence of eigenfunctions of the Feller pricing semigroup; see (3.2) below for a precise definition. We will provide additional properties of the eigenfunctions in Proposition 3.1 below. In particular, we shall present an elegant way to obtain the coefficients of the Sheffer polynomials $\left(\mathcal{L}_{n}\right)_{n \geq 0}$ by solving a system of linear equations. Moreover, from [4, Proposition 5.1 and Remark 5.2] it follows that the sequence $\left(\mathcal{V}_{n}\right)_{n \geq 0}$ is a sequence of co-eigenmeasures in the sense that for any $t \geq 0$ and $f \in \mathcal{P}_{\lambda}$ with $\lambda>-R_{F}$,

$$
\mathcal{V}_{n}\left(P_{t} f\right)=e^{-\lambda_{n} t} \mathcal{V}_{n} f
$$

The phenomenon that the expansion holds only for $T$ bigger than a nonnegative constant (namely $\underline{\mathrm{T}}$ ) has been observed in the framework of non-self-adjoint Schrödinger operators (see [5]) as well as for some non-self-adjoint Markov semigroups (see [32], [31], and [33]). This fact together with the previous remark illustrates a fundamental difference with self-adjoint Markov semigroups, for which the eigenfunctions and the density of co-eigenmeasures coincide and the expansion is valid for all $T>0$.

In order to apply Theorem 2.2 it is useful to know the values of $\underline{T}$ and $\bar{\lambda}_{T}$. If the branching mechanism is such that there is an analytic formula for $\bar{A}$ (as is the case in the examples of section 4), then one can determine $\underline{\mathrm{T}}$ (and consequently $\bar{\lambda}_{T}$ ) explicitly using (2.17)-(2.19) and $R_{F}=R_{\psi} \wedge R_{\phi} \wedge \underline{\theta}$ (see Lemma A.1). Otherwise, it seems difficult to determine analytically $\underline{\mathrm{T}}$ and one might need to resort to numerical methods for computing $\underline{T}$, which would in particular involve computing the radius of convergence of the power series $G_{x}$ in (2.18). It would be interesting to have sufficient conditions that imply $\underline{T}=0$ for a larger class of examples than the ones we consider in section 4. Karlin and McGregor [18, section 5] have given some results in this direction for Galton-Watson processes and we leave it for further research to see whether their ideas could be used in our setting.

2.2. Examples: Bond and yield options. The main feature of an affine term structure model is the exponential-affine dependence of the prices of the zero-coupon bonds with respect to the short rate. This allows us to use the short rate pricing semigroup for the pricing of 
options on bonds or on the yield and we obtain the series representation of these options values as a corollary of Theorem 2.2. First, recall from (2.9) that if we denote by $\Psi$ and $\Phi$ the CBI exponents of the pricing semigroup, then the price at time $T$ of an $S$-bond is given by

$$
B_{T}(S)=\mathbb{E}\left[e^{-\int_{T}^{S} r_{t} d t} \mid \mathcal{F}_{T}\right]=e^{-\Phi(\tau)-\Psi(\tau) r_{T}}
$$

where we recall that $\tau=S-T$ and, for any $t \geq 0, \Phi(t)=\Phi_{t}(0)$ and $\Psi(t)=\Psi_{t}(0)$. The yield to maturity $S$ at time $T \leq S$, denoted by $Y_{T}(S)$, takes the form

$$
Y_{T}(S)=\frac{-\ln B_{T}(S)}{\tau}=\frac{\Phi(\tau)}{\tau}+\frac{\Psi(\tau)}{\tau} r_{T}
$$

From (2.1), (2.31), and (2.32), respectively, the price today of a European option written on the maturity $S$-bond, with payoff function $g$ at time $T<S$, is given, for any $x \geq 0$, by

$$
\mathbb{E}_{x}\left[e^{-\int_{0}^{T} r_{t} d t} g\left(B_{T}(S)\right)\right]=P_{T} f_{B}(x) \text { where } f_{B}(x)=g\left(e^{-\Phi(\tau)-x \Psi(\tau)}\right),
$$

and the price today of a European option written on the yield to maturity $S$, expiring at time $T<S$, with payoff function $g$ at time $T$, is given, for any $x \geq 0$, by

$$
\mathbb{E}_{x}\left[e^{-\int_{0}^{T} r_{t} d t} g\left(Y_{T}(S)\right)\right]=P_{T} f_{Y}(x), \text { where } f_{Y}(x)=g\left(\frac{\Phi(\tau)+\Psi(\tau) x}{\tau}\right) .
$$

In particular, from Theorem 2.2, we get that a European call option on an $S$-bond, with strike $K$ and expiry date $T \in(\underline{\mathrm{T}}, S)$, has its price given by

$$
\operatorname{Call}(x, K, \tau, T):=P_{T} f_{\mathrm{BCall}}(x)=\sum_{n=0}^{\infty} e^{-\lambda_{n} T} \mathcal{L}_{n}^{\theta}(x) \mathcal{V}_{n}\left(f_{\mathrm{BCall}}\right),
$$

where $f_{\mathrm{BCall}}(x)=\left(e^{-\Phi(\tau)-\Psi(\tau) x}-K\right)_{+}$. Similarly, the price of a European put option on the yield to maturity $S$ with strike $K$ and expiry date $T \in(\underline{\mathrm{T}}, S)$ is given by

$$
\operatorname{Put}(x, K, \tau, T):=P_{T} f_{\mathrm{YPut}}(x)=\sum_{n=0}^{\infty} e^{-\lambda_{n} T} \mathcal{L}_{n}^{\theta}(x) \mathcal{V}_{n}\left(f_{\mathrm{YPut}}\right)
$$

with $f_{\mathrm{YPut}}(x)=\left(K-\frac{\Phi(\tau)}{\tau}-\frac{\Psi(\tau)}{\tau} x\right)_{+}$. Note that since $f_{\mathrm{BCall}}$ and $f_{\mathrm{YPut}}$ have compact supports, both payoffs lie in $\mathcal{P}_{\lambda}$ for any $\lambda \in \mathbb{R}$. Note that, here, we focus on pricing options on zerocoupon bonds; however, one can also get prices of options on (nonzero-) coupon bonds by applying Jamshidian's device [15], which expresses an option price for a coupon bond as a sum of option prices for zero-coupon bonds.

3. Numerical methods. In this part, we aim to give three methods for pricing some European options in a one-dimensional positive affine term structure model. We start by providing a way to compute the Sheffer polynomials $\left(\mathcal{L}_{n}\right)_{n \geq 0}$ by solving a system of linear equations, based on the fact that the functions $\left(\mathcal{L}_{n}^{\theta}\right)_{n \geq 0}$ are eigenfunctions of the pricing semigroup. We then present some Laplace-Fourier inversion techniques that will be useful for the computations of the sequence of integrals $\left(\mathcal{V}_{n} f\right)_{n \geq 0}$. 
3.1. Computation of the Sheffer polynomials $\left(\mathcal{L}_{n}\right)_{n \geq 0}$. One way to compute the polynomials $\mathcal{L}_{n}$ is via Taylor's formula by taking the $n$th derivative at zero of the right-hand side of (2.18). This requires that one knows $F$ and $\bar{A}$ and thus also $A$ explicitly, which is not always the case. Below, we provide an alternative way of computing the eigenfunctions which does not require any knowledge of $A, \bar{A}, F$ except for the quantity $1 / A^{\prime}(\theta)$, which by $(2.14)$ is given by

$$
\frac{1}{A^{\prime}(\theta)}=\int_{\theta}^{\infty} \exp \left(-\int_{\theta}^{v} \frac{\psi^{\prime}(u)-\psi^{\prime}(\theta)}{\psi(u)} d u\right) d v
$$

Proposition 3.1. Let $\left(\mathcal{L}_{n}\right)_{n \geq 0}$ be defined by (2.18).

1. The sequence $\left(\mathcal{L}_{n}^{\theta}\right)_{n \geq 0}$ is a sequence of eigenfunctions for the pricing semigroup $P$ associated with the sequence of eigenvalues $\left(e^{-\lambda_{n} t}\right)_{n \geq 0}$ in the sense that $\mathcal{L}_{n}^{\theta} \in C_{0}\left(\mathbb{R}_{\geq 0}^{+}\right)$ and for any $t, x \geq 0$,

$$
P_{t} \mathcal{L}_{n}^{\theta}(x)=e^{-\lambda_{n} t} \mathcal{L}_{n}^{\theta}(x)
$$

Moreover, for any $n \geq 0, \mathcal{L}_{n}^{\theta} \in \mathcal{D}$, the domain of the infinitesimal generator, and, for any $x \geq 0$, we have

$$
\mathcal{A L}_{n}^{\theta}(x)=-\lambda_{n} \mathcal{L}_{n}^{\theta}(x)
$$

where $\mathcal{A}$ is the infinitesimal generator of $P$.

2. With the notation $\mathcal{L}_{n}(x)=\sum_{k=0}^{n} a_{k, n} x^{k}, n \geq 0$, the coefficients $a_{k, n}$ are given by

$$
a_{n, n}=\frac{(-1)^{n}}{n !} \frac{1}{\left(A^{\prime}(\theta)\right)^{n}}
$$

and

$$
\left(a_{0, n} ; \ldots ; a_{n-1, n}\right)^{T}=\mathbf{B}^{-1} \mathbf{c},
$$

where, writing $\psi^{(k)}$ (resp., $\left.\phi^{(k)}\right)$ for the $k$ th derivative of $\psi$ (resp., $\left.\phi\right), \mathbf{c}=\left(\mathbf{c}_{j}\right)_{j=0}^{n-1}$ is the column vector given by

$$
\mathbf{c}_{j}= \begin{cases}(-1)^{n} a_{n, n} \phi^{(n)}(\theta), & j=0, \\
(-1)^{n-j} a_{n, n}\left(\left(\begin{array}{c}
n \\
j-1
\end{array}\right) \psi^{(n+1-j)}(\theta)+\left(\begin{array}{c}
n \\
j
\end{array}\right) \phi^{(n-j)}(\theta)\right), & j=1, \ldots, n-1,\end{cases}
$$

and $\mathbf{B}^{-1}$ is the inverse of the upper-triangular matrix $\mathbf{B}=\left(\mathbf{B}_{j, k}\right)_{j, k=0}^{n-1}$ given by

$$
\mathbf{B}_{j, k}= \begin{cases}(-1)^{k+1} \phi^{(k)}(\theta), & j=0 ; \quad k=1, \ldots, n-1, \\
(n-j) \psi^{\prime}(\theta), & j=0, \ldots, n-1 ; \quad k=j, \\
(-1)^{k+1-j}\left(\left(\begin{array}{c}
k \\
j-1
\end{array}\right) \psi^{(k+1-j)}(\theta)+\left(\begin{array}{c}
k \\
j
\end{array}\right) \phi^{(k-j)}(\theta)\right), & j=1, \ldots, n-1 ; \\
0 & k=j+1, \ldots, n-1, \\
& \text { otherwise. }\end{cases}
$$

Copyright $($ ) by SIAM. Unauthorized reproduction of this article is prohibited. 
3.2. Computation of the coefficients $\left(\mathcal{V}_{n} f\right)_{n \geq 0}$ via Laplace-Fourier inversion techniques. The second main ingredient appearing in the eigenvalues expansion (2.28) of the pricing semigroup is the sequence $\left(\mathcal{V}_{n} f\right)_{n \geq 0}$, which is well-defined for $f \in \cup_{\lambda>-R_{F}} \mathcal{P}_{\lambda}$; see Lemma A.3 below. For some specific combination of mechanisms and payoffs $f$ such expression may be computed explicitly; see, e.g., section 4.1. Otherwise, we suggest an alternative methodology which is based on Laplace-Fourier inversion techniques. We focus here on the type of payoffs that appear in the options prices (2.35) and (2.36). Note that in these option prices, integrals appear of the form

$$
\int_{0}^{\infty} h(k, y) \mathcal{V}(d y)
$$

where $\mathcal{V}$ is a (signed) measure on $\mathbb{R}^{+}$with Laplace transform $\widehat{\mathcal{V}}(\lambda)=\int_{0}^{\infty} e^{-\lambda y} \mathcal{V}(d y)$ which exists for $\lambda>\lambda_{0}$ for some $\lambda_{0} \in \mathbb{R}$ and where $h(k, y), y, k \geq 0$, is a kernel of the form $h(k, y)=\left(e^{-(a+b y)}-e^{-k}\right)_{+}$or $h(k, y)=(k-(a+b y))_{+}$with $a \in \mathbb{R}$ and $b>0$. One can get an expression for the Laplace transform in $k$ of (3.6) in terms of $\widehat{\mathcal{V}}$. Consequently one can compute (3.6) numerically by performing one Laplace inversion. This idea has been worked out in the works of, e.g., Duffie, Pan, and Singelton [10], Carr and Madan [3], and Lee [24]. The next proposition can be extracted from those references and we provide its proof in the appendix for convenience.

Proposition 3.2. Let $\rho>\frac{\lambda_{0}}{b} \vee 0$. Then

$$
\begin{aligned}
\int_{0}^{\infty}\left(e^{-(a+b y)}-e^{-k}\right)_{+} \mathcal{V}(d y) & =e^{\rho k} \frac{1}{2 \pi} \int_{-\infty}^{\infty} e^{\mathrm{i} u k} \frac{e^{-(1+\rho+\mathrm{i} u) a} \widehat{\mathcal{V}}(b(1+\rho+\mathrm{i} u))}{(\rho+\mathrm{i} u)(1+\rho+\mathrm{i} u)} d u \\
\int_{0}^{\infty}(k-(a+b y))_{+} \mathcal{V}(d y) & =e^{\rho k} \frac{1}{2 \pi} \int_{-\infty}^{\infty} e^{\mathrm{i} u k} \frac{e^{-(\rho+\mathrm{i} u) a} \widehat{\mathcal{V}}(b(\rho+\mathrm{i} u))}{(\rho+\mathrm{i} u)^{2}} d u
\end{aligned}
$$

3.3. Description of the numerical methods. For the numerical computation of the option prices Call $(x, K, \tau, T)$ and $\operatorname{Put}(x, K, \tau, T)$ defined in (2.35) and (2.36), we distinguish between the following three methods:

1. The Laplace method. One uses (3.7) with $\mathcal{V}(d y)=P_{T}(x, d y)$ in combination with (2.33) and (2.34) to compute the option prices via one Laplace inversion. Recall here that the Laplace transform of $P_{T}(x, d y)$ is given by $(2.2)$.

2. The explicit-spectral method. In this case, one has closed-form expressions for the sequence of co-eigenmeasures $\left(\mathcal{V}_{n}\right)_{n>0}$ that lead to explicit expressions of the integrals $\int_{0}^{\infty} h(k, y) \mathcal{V}_{n}(d y)$. Thus, one can use (2.35) and (2.36) to compute the option prices by truncating the infinite sum.

3. The spectral-Laplace method. One uses (2.35) and (2.36) by truncating the infinite sum and computing each integral in the sum via a Laplace inversion using (3.7) with $\mathcal{V}=\mathcal{V}_{n}$. For this method one needs to know the Laplace transform of $\mathcal{V}_{n}$, which equals $\widehat{\mathcal{V}}_{n}(\lambda)=e^{-F(\lambda)} A(\lambda)^{n}$; see Lemma A.3 below.

Note that the Laplace method is a well-known method for computing the option prices in an affine short rate model and due to its speed and simplicity should be preferred over methods involving Monte Carlo or (partial) integro-differential equations. The contribution of

Copyright (C) by SIAM. Unauthorized reproduction of this article is prohibited. 
this section is to propose the two other methods, based on the spectral expansion approach, as alternatives and compare them with the Laplace method. The quality of a numerical method depends on its accuracy and its speed. While in the two numerical experiments in section 5.1 we will see how accurately one can compute the option prices Call $(x, K, \tau, T)$ and $\operatorname{Put}(x, K, \tau, T)$ via the three methods and in particular how many terms in the sum in $(2.35)$ and (2.36) are needed for the two spectral methods to get a close approximation of the option prices, we now discuss some pros and cons of the three methods which can readily be observed from their description. These points will be further illustrated in section 5.2, where we look at the computation times of the methods.

The explicit-spectral method has the advantage that it is very explicit and thus fast. In particular it involves no Laplace inversion. Its downside is that it cannot be applied when one cannot find explicit analytic expressions for the co-eigenmeasures. The major advantage of both spectral methods is that it is very cheap to compute option prices for a range of increasing expiry times $T$ (thereby keeping $\tau=S-T$ fixed), since the integrals (as well as the eigenfunctions $\mathcal{L}_{n}^{\theta}$ ) in the formulas in (2.35) and (2.36) do not depend on $T$ and by Theorem $2.2(3)$ the larger $T$, the faster the convergence. Hence with the spectral methods, if one has computed an option price for the expiry time $T$, it takes almost no extra computational time to get accurate option prices for any expiry time larger than $T$. This is in contrast to the Laplace method where one has to redo the Laplace inversion if $T$ alters. However, the Laplace method has the advantage that for a single expiry time $T$, only one Laplace inversion needs to be done, whereas for the spectral-Laplace method one needs to do a Laplace inversion for each term in the sum in (2.35) and (2.36).

Numerical methods to perform a Laplace inversion typically involve computing the Laplace transform on a grid of (complex-valued) points. Although, for the spectral-Laplace method, one needs to perform a Laplace inversion for each term in the sum in (2.35) and (2.36), it is very cheap to compute the necessary Laplace transforms corresponding to one extra term in the sum due to the special product structure in $n$ of the Laplace transform $\widehat{\mathcal{V}}_{n}$ of the coeigenmeasures $\mathcal{V}_{n}$, namely, $\frac{\widehat{\mathcal{V}}_{n+1}(\lambda)}{\widehat{\mathcal{V}}_{n}(\lambda)}=A(\lambda)$ (see Lemma A.3), which leads to the same simple product structure of the Laplace transform of the integrals in (3.7) with $\mathcal{V}=\mathcal{V}_{n}$.

The two Laplace methods work especially well when there exist explicit expressions for all the Laplace transforms involved (i.e., $A$ and $F$ for the spectral-Laplace method and $\Psi_{t}$ and $\Phi_{t}$ for the Laplace method), since then these Laplace transforms can be quickly evaluated. However, this is not always the case and then one has to numerically compute the Laplace transforms as well and as a consequence the computational time of the two Laplace methods will be dominated by the time it takes to numerically compute these transforms. In this case the spectral-Laplace method has an additional big advantage over the Laplace method. Namely, numerically solving the ODEs (2.3)-(2.4) gives $\Psi_{t}(\lambda)$ and $\Phi_{t}(\lambda)$ on a $t$-grid for one value of $\lambda$ (the initial condition) and so one needs to compute these ODEs many times over for different values of $\lambda$ before being able to perform the Laplace inversion. Instead with the spectral-Laplace method one needs to numerically solve the two corresponding transforms $A$ and $F$ only once and one has the required Laplace transforms $\widehat{\mathcal{V}}_{n}(\lambda), n=0,1,2, \ldots$, directly on a $\lambda$-grid. Moreover, in the spectral-Laplace method $A$ and $F$ can be computed independently from each other (and therefore a speed-up can be obtained via parallel computing), whereas in the Laplace method such a trick is not possible, since $\Phi_{t}(\lambda)$ depends on $\Psi_{t}(\lambda)$.

Copyright (c) by SIAM. Unauthorized reproduction of this article is prohibited. 
We stress that the application of the two spectral methods (and of course also the Laplace method) is not restricted to computing merely the option prices Call $(x, K, \tau, T)$ and $\operatorname{Put}(x, K$, $\tau, T$ ), but that the methods (after obvious modifications) can potentially be used as well for pricing other options and, more generally, for any other application that involves the transition distribution of a CBI-process.

4. Examples of expansions. In this section we provide two examples where at least part of the ingredients of the spectral decomposition of the pricing semigroup can be obtained more explicitly, which as a result yields more explicit expressions for the prices for the European bond call option and the European yield put option.

4.1. Around self-similar CBI-processes. We consider the CBI-process where the mechanisms are given, for any $u \geq 0$, by

$$
\begin{aligned}
& \psi(u)=a(u+\eta)^{\alpha+1}-b(u+\eta), \\
& \phi(u)=a c(u+\eta)^{\alpha}-q,
\end{aligned}
$$

with parameters $a>0, \eta>0, c>0,0<\alpha \leq 1, b=\frac{1+a \eta^{\alpha+1}}{\eta}$, which ensures $\psi(0)=-1$ and $q=a c \eta^{\alpha}$, which ensures $\phi(0)=0$. When $\alpha=1$ the above mechanisms correspond to the CIR model. If $\alpha<1$, the paths of the CBI-process contain jumps and the corresponding Lévy measures $\Pi$ and $\mu$ in $(2.5)-(2.6)$ are given, for $y>0$, by

$$
\Pi(d y)=\frac{a \alpha(\alpha+1)}{\Gamma(1-\alpha)} \mathrm{e}^{-\eta y} y^{-\alpha-2} d y, \quad \mu(d y)=\frac{c a \alpha}{\Gamma(1-\alpha)} \mathrm{e}^{-\eta y} y^{-\alpha-1} d y
$$

where $\Gamma(z)=\int_{0}^{\infty} t^{z-1} \mathrm{e}^{-t} d t, \mathfrak{R e}(z)>0$, is the gamma function. Since $\int_{1}^{\infty} y \Pi(d y)<\infty$, it follows that condition (2.8) is satisfied. For this example (2.7) reads as

$$
\mathcal{A} f(x)= \begin{cases}a x f^{\prime \prime}(x)+(a c+(1 / \eta-a \eta) x) f^{\prime}(x)-x f(x) & \text { if } \alpha=1, \\ \left(1 / \eta-a \alpha \eta^{\alpha}\right) x f^{\prime}(x)-x f(x) & \\ +x \int_{0}^{\infty}\left(f(x+y)-f(x)-f^{\prime}(x) y\right) \Pi(d y)+\int_{0}^{\infty}(f(x+y)-f(x)) \mu(d y) & \text { if } \alpha<1 .\end{cases}
$$

We note that this example has also been treated in Ogura [29] and that when $\eta=q=0$, the CBI-process is self-similar; see [30] for a detailed study of this family. We will now give some explicit expressions for the various quantities defined earlier in section 2 . We easily see that the branching mechanism satisfies condition (2.10) and the positive root of $\psi$ is given by $\theta=\left(\frac{b}{a}\right)^{\frac{1}{\alpha}}-\eta$ and $\psi^{\prime}(\theta)=b \alpha$. Hence by Theorem 2.2, the pricing semigroup exhibits the spectral representation (2.28). In order to identify the various ingredients, first note that one can easily check that the functions $A, \bar{A}$, and $F$ are given by

$$
A(\lambda)=1-\left(\frac{\theta+\eta}{\lambda+\eta}\right)^{\alpha}, \quad \bar{A}(u)=(\theta+\eta)(1-u)^{-1 / \alpha}-\eta, \quad e^{-F(\lambda)}=\left(\frac{\theta+\eta}{\lambda+\eta}\right)^{c} .
$$

Copyright (C) by SIAM. Unauthorized reproduction of this article is prohibited. 
Note that $R_{F}=R_{A}=\eta$ and $R_{\bar{A}}=1$. Then by (2.15) and (2.16),

$$
\begin{aligned}
\Psi_{t}(\lambda) & =(\theta+\eta)\left(1-e^{-b \alpha t}\left[1-\left(\frac{\theta+\eta}{\lambda+\eta}\right)^{\alpha}\right]\right)^{-1 / \alpha}-\eta \\
e^{-\Phi_{t}(\lambda)} & =e^{-(c b-q) t}\left(\frac{\theta+\eta}{\lambda+\eta}\right)^{c}\left(1-e^{-b \alpha t}\left(1-\left(\frac{\theta+\eta}{\lambda+\eta}\right)^{\alpha}\right)\right)^{-c / \alpha} .
\end{aligned}
$$

Next, we compute the components of the spectral representation. In order to get the expression of the co-eigendensities $\nu_{n}$, as defined in (2.25) (note that $F(\infty)=\infty$ ), recalling that

$$
(\lambda+\eta)^{-z}=\frac{1}{\Gamma(z)} \int_{0}^{\infty} \mathrm{e}^{-\lambda y} \mathrm{e}^{-\eta y} y^{z-1} \mathrm{~d} y, \quad \mathfrak{R e}(z)>0
$$

we deduce by (2.22) and (2.23) that

Using that

$$
\nu(y)=\frac{(\theta+\eta)^{c}}{\Gamma(c)} \mathrm{e}^{-\eta y} y^{c-1} \quad \text { and } \quad \omega(y)=\frac{(\theta+\eta)^{\alpha}}{\Gamma(\alpha)} \mathrm{e}^{-\eta y} y^{\alpha-1} .
$$

$$
\int_{0}^{y} e^{-\eta(y-z)} \frac{(y-z)^{\gamma-1}}{\Gamma(\gamma)} \mathrm{e}^{-\eta z} \frac{z^{\beta-1}}{\Gamma(\beta)} d z=e^{-\eta y} \frac{y^{\gamma+\beta-1}}{\Gamma(\gamma+\beta)},
$$

we get by (2.24) and (2.25) that

$$
\begin{aligned}
\mathcal{W}_{n}(y) & =\sum_{j=1}^{n}\left(\begin{array}{c}
n \\
j
\end{array}\right)(-1)^{j} \omega^{* j}(y)=\mathrm{e}^{-\eta y} \sum_{j=1}^{n}(-1)^{j}\left(\begin{array}{c}
n \\
j
\end{array}\right) \frac{(\theta+\eta)^{\alpha j}}{\Gamma(\alpha j)} y^{\alpha j-1}, \\
\nu_{n}(y) & =(\theta+\eta)^{c} \mathrm{e}^{-\eta y} y^{c-1} \sum_{j=0}^{n}(-1)^{j}\left(\begin{array}{c}
n \\
j
\end{array}\right) \frac{((\theta+\eta) y)^{\alpha j}}{\Gamma(c+\alpha j)} .
\end{aligned}
$$

The eigenfunctions can be computed via (2.18) or Proposition 3.1 and we do not give any further details here. The eigenvalues are given by

$$
\lambda_{n}=b \alpha n+c b-q
$$

see (2.26). Further, the value $R_{G}$ defined in (2.18) is equal to 1 , since $R_{\bar{A}}=1$ and $R_{F \circ \bar{A}}=1$. From this it follows that $\underline{\mathrm{T}}=0$ in (2.19).

In order to compute the option prices (2.33) and (2.34) via the explicit-spectral method, we need to have explicit expressions for the integrals $\mathcal{V}_{n}\left(f_{B \text { Call }}\right)$ and $\mathcal{V}_{n}\left(f_{Y \text { Put }}\right)$. Denoting by $\gamma(z, x)=\int_{0}^{x} t^{z-1} \mathrm{e}^{-t} \mathrm{~d} t, \mathfrak{R e}(z)>0$, the lower incomplete gamma function and recalling the recurrence relation $\gamma(z+1, x)=z \gamma(z, x)-x^{z} \mathrm{e}^{-x}$, we get, for any $n \geq 0$,

$$
\begin{aligned}
\mathcal{V}_{n}\left(f_{\text {BCall }}\right) & =\int_{0}^{\infty}\left(\mathrm{e}^{-\Phi(\tau)-\Psi(\tau) y}-K\right)_{+} \nu_{n}(y) d y \\
& =(\theta+\eta)^{c} \sum_{j=0}^{n} \frac{(-1)^{j}\left(\begin{array}{c}
n \\
j
\end{array}\right)\left(\frac{b}{a}\right)^{j}}{\Gamma(c+\alpha j)} C_{B}(j, K, \tau) \mathbf{1}_{\{\Phi(\tau) \leq-\ln K\}}, \\
\mathcal{V}_{n}\left(f_{\mathrm{YPut}}\right) & =\int_{0}^{\infty}\left(K-\frac{\Phi(\tau)}{\tau}-\frac{\Psi(\tau)}{\tau} y\right)_{+} \nu_{n}(y) d y \\
& =(\theta+\eta)^{c} \sum_{j=0}^{n} \frac{(-1)^{j}\left(\begin{array}{c}
n \\
j
\end{array}\right)\left(\frac{b}{a}\right)^{j}}{\Gamma(c+\alpha j)} C_{Y}(j, K, \tau) \mathbf{1}_{\{\Phi(\tau) \leq K \tau\}}
\end{aligned}
$$

Copyright (C) by SIAM. Unauthorized reproduction of this article is prohibited. 
with

$$
\begin{aligned}
C_{B}(j, K, \tau)= & \left(\mathrm{e}^{-\Phi(\tau)} \frac{\gamma\left(c+\alpha j,(\eta+\Psi(\tau)) \frac{-\ln K-\Phi(\tau)}{\Psi(\tau)}\right)}{(\eta+\Psi(\tau))^{c+\alpha j}}-K \frac{\gamma\left(c+\alpha j, \eta \frac{-\ln K-\Phi(\tau)}{\Psi(\tau)}\right)}{\eta^{c+\alpha j}}\right), \\
C_{Y}(j, K, \tau)= & \left(K-\frac{\Phi(\tau)}{\tau}-\frac{\Psi(\tau)}{\tau \eta}(c+\alpha j)\right) \frac{\gamma\left(c+\alpha j, \eta \frac{K \tau-\Phi(\tau)}{\Psi(\tau)}\right)}{\eta^{c+\alpha j}} \\
& +\frac{\Psi(\tau)}{\tau \eta}\left(\frac{K \tau-\Phi(\tau)}{\Psi(\tau)}\right)^{c+\alpha j} \mathrm{e}^{-\eta \frac{K \tau-\Phi(\tau)}{\Psi(\tau)}} .
\end{aligned}
$$

We now have all the ingredients to compute the option prices (2.33) and (2.34) by any of the three methods described in section 3.3.

4.2. A CIR model with jumps. We shall now consider the CIR model with jumps which was introduced by [9] for financial applications. Let the pricing semigroup $\left(P_{t}\right)_{t \geq 0}$ be characterized by the following branching and immigration mechanisms:

$$
\begin{aligned}
& \psi(u)=\sigma^{2} u^{2}+b u-1, \\
& \phi(u)=\sigma^{2} c u+\sigma^{2} \frac{p u}{q(u+q)}
\end{aligned}
$$

with parameters $b, c, p \geq 0$ and $\sigma^{2}, q>0$. Note that the measure $\mu(d y)$ in (2.6) is given by

$$
\mu(d y)=\sigma^{2} p \mathrm{e}^{-q y} d y, \quad y>0 .
$$

For this example (2.7) can be written as

$$
\mathcal{A} f(x)=\sigma^{2} x f^{\prime \prime}(x)+\left(\sigma^{2} c-b x\right) f^{\prime}(x)-x f(x)+\int_{0}^{\infty}(f(x+y)-f(x)) \mu(d y) .
$$

We have

$$
\theta=\left(-b+\sqrt{b^{2}+4 \sigma^{2}}\right) / 2 \sigma^{2}, \quad \underline{\theta}=\left(b+\sqrt{b^{2}+4 \sigma^{2}}\right) / 2 \sigma^{2}
$$

and we set $\delta=\theta+\underline{\theta}=\sqrt{b^{2}+4 \sigma^{2}} / \sigma^{2}$. Furthermore as the condition (2.10) is satisfied, one may apply Theorem 2.2. In the next lemma, we give all the necessary ingredients to compute the option prices of section 2.2 with the two Laplace methods. Note that the eigenfunctions can be computed either via (2.18) or by means of Proposition 3.1.

Lemma 4.1. Let $g(t)=\frac{1-e^{-\sigma^{2} \delta t}}{\delta}$ for any $t \geq 0$. Then, the CBI exponents associated with the mechanisms (4.3) are given by

$$
\Psi_{t}(\lambda)=\frac{\delta}{1-e^{-\sigma^{2} \delta t}\left(1-\frac{\delta}{\lambda+\underline{\theta}}\right)}-\underline{\theta}
$$

and

$$
\mathrm{e}^{-\Phi_{t}(\lambda)}= \begin{cases}\mathrm{e}^{-\phi(\theta) t}(1+g(t)(\lambda-\theta))^{-c}\left(1+g(t)(\lambda-\theta) \frac{\theta-\delta+q}{\lambda+q}\right)^{-\frac{\sigma^{2} p}{\psi(-q)}} & \text { if } q \neq \underline{\theta}, \\ \mathrm{e}^{-\phi(\theta) t}(1+g(t)(\lambda-\theta))^{-c} \exp \left(-g(t)(\lambda-\theta) \frac{p}{\delta(\lambda+\underline{\theta})}\right) & \text { if } q=\underline{\theta}\end{cases}
$$


Furthermore,

$$
A(\lambda)=1-\frac{\delta}{\lambda+\underline{\theta}}, \quad \bar{A}(u)=\frac{\delta}{1-u}-\underline{\theta}
$$

and

$$
e^{-F(\lambda)}= \begin{cases}\Lambda_{\theta}(\lambda+\underline{\theta})^{-c}\left(\frac{\lambda+q}{\lambda+\underline{\theta}}\right)^{\frac{\sigma^{2} p}{\psi(-q)}} & \text { if } q \neq \underline{\theta}, \\ \Lambda_{\theta}(\lambda+\underline{\theta})^{-c} \exp \left(\frac{p}{\delta(\lambda+\underline{\theta})}\right) & \text { if } q=\underline{\theta},\end{cases}
$$

where $\Lambda_{\theta}$ is the positive constant such that $e^{-F(\theta)}=1$. Finally, $\underline{\mathrm{T}}=0$.

Proof. We have

$$
-\frac{\psi^{\prime}(\theta)}{\psi(\lambda)}=-\frac{\sigma^{2}(\theta+\underline{\theta})}{\sigma^{2}(\lambda-\theta)(\lambda+\underline{\theta})}=\frac{1}{\lambda+\underline{\theta}}-\frac{1}{\lambda-\theta}=-\frac{\sigma^{2} \delta}{\sigma^{2} \lambda(\lambda+\delta)}=\frac{1}{\lambda}-\frac{1}{\lambda+\delta}
$$

from which the formula for $A$ directly follows via (2.11). Now the formula for $\bar{A}$ which is the inverse of $A$ follows as well. Then via (2.15), we get $\Psi_{t}(\lambda)$. Next, observe that, for $\lambda \neq \theta$,

$$
\begin{aligned}
-\frac{\phi(\lambda)-\phi(\theta)}{\psi(\lambda)} & =-\frac{\lambda-\theta}{\psi(\lambda)}\left(c \sigma^{2}+\frac{\sigma^{2} p}{(\lambda+q)(\theta+q)}\right) \\
& =-\frac{c}{\lambda+\underline{\theta}}-\frac{p}{(\lambda+\underline{\theta})(\lambda+q)(\theta+q)}
\end{aligned}
$$

and so it follows that, for $\lambda \neq \theta$,

$$
-\frac{\phi(\lambda)-\phi(\theta)}{\psi(\lambda)}= \begin{cases}-c(\lambda+\underline{\theta})^{-1}+\frac{\sigma^{2} p}{\psi(-q)}\left((\lambda+q)^{-1}-(\lambda+\underline{\theta})^{-1}\right) & \text { if } q \neq \underline{\theta}, \\ -c(\lambda+\underline{\theta})^{-1}-\frac{p}{\delta}(\lambda+\underline{\theta})^{-2} & \text { if } q=\underline{\theta} .\end{cases}
$$

This yields the formula for $e^{-F(\lambda)}$ via (2.12). Since, in addition, one can easily check that

$$
\frac{\lambda+\underline{\theta}}{\Psi_{t}(\lambda)+\underline{\theta}}=1+g(t)(\lambda-\theta)
$$

and in the case where $q \neq \underline{\theta}$,

$$
\frac{\Psi_{t}(\lambda)+q}{\lambda+q}=\frac{1+g(t)(\lambda-\theta) \frac{\theta-\delta+q}{\lambda+q}}{1+g(t)(\lambda-\theta)}
$$

we conclude via (2.16) that the desired formula for $\Phi_{t}(\lambda)$ holds in the case where $q \neq \underline{\theta}$. Noting that (4.4) implies

$$
\frac{1}{\lambda+\underline{\theta}}-\frac{1}{\Psi_{t}(\lambda)+\underline{\theta}}=-g(t) \frac{\lambda-\theta}{\lambda+\underline{\theta}},
$$

we also see that the formula for $\Phi_{t}(\lambda)$ is correct in the case where $q=\underline{\theta}$.

Finally, since $\bar{A}(u)$ is analytic in $(-1,1), \mathfrak{R e}(\bar{A}(z))>-\underline{\theta}$ for $|z|<1$ and $F(z)$ is analytic for $\mathfrak{R e}(z)>-\underline{\theta}$ (see Lemma A.1), it follows that $R_{G}=1$ and $\underline{\mathrm{T}}=0$.

Remark 4.2. Although it is possible to get an expression involving a power series in $y$ for the co-eigendensities $\left(\nu_{n}(y)\right)_{n \geq 0}$ (note that $F(\infty)=\infty$ ) by inverting its Laplace transform, it is very hard to evaluate it since this power series has coefficients which alternate in sign. For this model, we therefore recommend avoiding the explicit-spectral method and using the spectral-Laplace method instead. 
5. Numerical experiments. In this section we perform some numerical experiments in order to give an illustration of the efficiency of the numerical methods described in section 3 . We will in particular look at the spectral error (i.e., the error that is caused by truncating the infinite sum in the spectral expansion) and the computation times of the methods.

5.1. Spectral error. We will determine, for a range of expiry times $T$ and strikes $K$, how many terms of the infinite sum in (2.35) and (2.36) are needed to produce a spectral error that is lower than some threshold value. For this purpose, we introduce for $N \in\{1,2, \ldots\} \cup\{\infty\}$, the $N$ th order spectral approximation for the option price Call $(x, K, \tau, T)$ or $\operatorname{Put}(x, K, \tau, T)$,

$$
\mathcal{S}_{N}=\mathcal{S}_{N}(K, T)=e^{-\phi(\theta) T} \sum_{n=0}^{N-1} e^{-n \psi^{\prime}(\theta) T} \mathcal{L}_{n}^{\theta}(x) \int_{0}^{\infty} f(K, y) \mathcal{V}_{n}(d y)
$$

with $f$ the payoff kernel corresponding to the particular option. Theorem 2.2(3) says that, for any $T>\underline{T}, \mathcal{S}_{N}$ converges exponentially to $\mathcal{S}_{\infty}$ as $N \rightarrow \infty$ and at a rate that decreases to 0 monotonically as $T \downarrow \underline{T}$. In order to see how fast the convergence is in practice, we introduce for $\epsilon>0$,

$$
\mathcal{N}_{\epsilon}=\inf \left\{N \geq 4: \max \left\{\left|\mathcal{S}_{N}-\mathcal{S}_{N-1}\right|,\left|\mathcal{S}_{N}-\mathcal{S}_{N-2}\right|,\left|\mathcal{S}_{N}-\mathcal{S}_{N-3}\right|\right\} \leq \epsilon\left|\mathcal{S}_{N}\right|\right\} .
$$

The quantity $\mathcal{N}_{\epsilon}$ is the lowest number of terms needed in the spectral expansion such that the spectral approximations have 'converged' in the sense that the relative errors of the $\left(\mathcal{N}_{\epsilon}-1\right)$ th, $\left(\mathcal{N}_{\epsilon}-2\right)$ th, and $\left(\mathcal{N}_{\epsilon}-3\right)$ th order spectral approximations, in comparison to the $\mathcal{N}_{\epsilon}$ th order spectral approximation, all do not exceed $\epsilon$. For the first experiments, we compute the numbers $\mathcal{N}_{\epsilon}$, where the spectral approximations $\mathcal{S}_{N}$ for $N=1,2, \ldots$ are computed by a spectral method.

Next we specify which option prices we compute. Namely, we compute the option price Call $(x, K, \tau, T)$ in the model with mechanisms (4.1), where the parameters chosen in (4.1) are

$$
\alpha=0.5, \quad a=1.0, \quad \eta=3.0, \quad c=2.5,
$$

and we compute the option price $\operatorname{Put}(x, K, \tau, T)$ for the model with mechanisms (4.3), where the chosen parameters are

$$
\sigma^{2}=1.0, \quad b=0.5, \quad c=1.5, \quad p=2.0, \quad q=3.0 .
$$

We compute the option prices for various strikes $K$ and expiry times $T$, whereas we fix $\tau=2$ and $x=0.05$. Here we take several strikes around the at-the-money level and two more extreme strikes corresponding to deep in-the-money options. Note that $\operatorname{Call}(x, K, \tau, T)=0$ if $K \geq e^{-\Phi(\tau)}$ and $\operatorname{Put}(x, K, \tau, T)=0$ if $K \leq \Phi(\tau) / \tau$. We therefore call $e^{-\Phi(\tau)}(\operatorname{resp} ., \Phi(\tau) / \tau)$ the zero-strike in the case of the bond call (resp., yield put). In order to avoid very small prices, all the options prices are multiplied by a factor 100, so that the resulting option prices correspond to options with notional value 100. In particular, for the calculation of $\mathcal{N}_{\epsilon}$, we compute the spectral approximations $\mathcal{S}_{N}$ in (5.1) with $f(K, y)=100\left(e^{-\Phi(\tau)-\Psi(\tau) y}-K\right)_{+}$in the bond call case and with $f(K, y)=100\left(K-\frac{\Phi(\tau)}{\tau}-\frac{\Psi(\tau)}{\tau} y\right)_{+}$in the yield put case. For the numerical Laplace inversions that are needed in the Laplace methods (i.e., the computation of the integrals in (3.7)), we make use of Filon's method [12]. For details on this particular 
method, we refer to section 5.2 of Kuznetsov, Kyprianou, and Rivero [22], where a clear and detailed description is given. Note that, as described in [22], one can combine Filon's method with the fast Fourier transform so that, as in [3], one can simultaneously compute option prices for a whole range of strikes. The error that is introduced by numerically inverting the Laplace transform is controlled by the number of discretization points chosen (i.e., the number $2 N$ in (114) of [22]) and the (uniform) distance between them (i.e., the parameter $h$ in (114) of [22]). For our numerical experiments we choose the number of discretization points to be $2^{14}=16,384$ in both examples and the distance between them to be $\frac{\pi}{0.00625 \cdot 2^{13}} \approx 0.06$ and $\frac{\pi}{0.01 \cdot 2^{13}} \approx 0.04$ for, respectively, the example with the bond call and the yield put option. Further we choose the damping parameter $\rho$ in (3.7) to be $\rho=\theta$ for both examples, where we recall that $\theta$ is the positive root of the branching mechanism $\psi$. With these choices the resulting (relative) error caused by the Fourier inversion is within $0.1 \%$ for all option prices.

In Tables 1 and 3 the value of $\mathcal{N}_{\epsilon}$ for $\epsilon=0.01$ and $\epsilon=0.001$ is computed for the two examples for various strikes and expiry times. The spectral approximations $\mathcal{S}_{N}$ are computed by the explicit-spectral method in Table 1 and by the spectral-Laplace method in Table 3 . We remark that we also ran a version of the first experiment where the spectral approximations are computed by the spectral-Laplace method and the results were identical. Further, in Tables 2 and 4, the corresponding spectral errors $\left|\frac{\mathcal{S}_{\mathcal{N}_{\epsilon}}-\mathcal{S}_{\infty}}{\mathcal{S}_{\infty}}\right|$ are computed; here the exact option prices $\mathcal{S}_{\infty}$ (see the definition in (5.1)) are computed via the Laplace method. For convenience the corresponding option prices computed by the Laplace method are displayed in Tables 5 and 6. All the computations were done in R [34]. From the numbers in Tables 2 and 4, we see that all the option prices can be accurately computed by the spectral methods except for the one with the highest strike and shortest maturity in Table 1. In line with Theorem 2.2(3), we see from Tables 1 and 3 that the spectral methods perform better as the expiry time increases in the sense that fewer terms in the sum in (2.35) and (2.36) are needed. When fixing $T$ and looking at the numbers $\mathcal{N}_{\epsilon}$ for different strikes $K$, we see that fewer terms are needed the more the option is in the money, i.e., the higher the option price. A possible explanation for this is that we have opted to work with relative errors. Coming back to the bond call option price for $T=1 / 12$ and $-\log K=0.925$, we see from Table 1 that the spectral approximations do not converge (at the $0.1 \%$ level). Although theoretically convergence should take place also in this case, the spectral sum typically consists of terms of both positive and negative sign which can

Table 1

Values of $\mathcal{N}_{0.01}$, respectively, $\mathcal{N}_{0.001}$, given on the left, respectively, right, side, of the columns for various $K$ and $T$ corresponding to the option price $100 \cdot$ Call $(0.05, K, 2, T)$ in the model with mechanisms (4.1) and parameter values (5.2); at-the-money-strike: $100 \mathrm{~K}=38.2744$; zero-strike: $100 \mathrm{~K}=40.3679$. The spectral approximations $\mathcal{S}_{N}, N=1,2, \ldots$, are computed by the explicit-spectral method.

\begin{tabular}{|cc||cc|cc|cc|cc|cc|cc|}
\hline \multicolumn{3}{|c|}{$\begin{array}{c}T \\
100 K\end{array}$} & \multicolumn{1}{|c|}{$1 / 12$} & & $2 / 12$ & & $3 / 12$ & $6 / 12$ & & 1 & & 2 \\
\hline \hline 39.6531 & 0.925 & 41 & $\infty$ & 35 & 39 & 24 & 34 & 13 & 21 & 10 & 12 & 7 & 8 \\
38.6741 & 0.950 & 24 & 40 & 22 & 25 & 20 & 24 & 12 & 18 & 10 & 12 & 7 & 8 \\
37.7192 & 0.975 & 20 & 34 & 18 & 20 & 13 & 20 & 12 & 13 & 10 & 11 & 7 & 8 \\
36.7879 & 1.000 & 13 & 26 & 13 & 24 & 13 & 17 & 12 & 13 & 9 & 11 & 7 & 8 \\
30.1194 & 1.200 & 12 & 14 & 12 & 13 & 9 & 13 & 9 & 12 & 8 & 9 & 6 & 7 \\
22.3130 & 1.500 & 10 & 13 & 10 & 13 & 10 & 12 & 8 & 10 & 6 & 8 & 6 & 6 \\
\hline
\end{tabular}

Copyright $\odot$ by SIAM. Unauthorized reproduction of this article is prohibited. 
Table 2

This table displays the relative spectral errors $\left|\frac{\mathcal{S}_{\mathcal{N}_{\epsilon}}-\mathcal{S}_{\infty}}{\mathcal{S}_{\infty}}\right| \times 100$ (written as a percentage and rounded off to 2 decimal places) for various $K$ and $T$ associated with the option price $100 \cdot$ Call $(0.05, K, 2, T)$ for two values of $\epsilon$, namely, $\epsilon=0.01=1 \%$ and $\epsilon=0.001=0.1 \%$ given on the left, respectively, right, side, of the columns, in the model with mechanisms (4.1) and parameter values (5.2). The values of $\mathcal{N}_{0.01}$ and $\mathcal{N}_{0.001}$ are displayed in Table 1. Here $\mathcal{S}_{\mathcal{N}_{\epsilon}}$, respectively, $\mathcal{S}_{\infty}$, is computed by the explicit-spectral method, respectively, Laplace method.

\begin{tabular}{|cc||cc|cc|cc|cc|cc|cc|c|}
\hline \multicolumn{3}{|c|}{$\begin{array}{c}T \\
100 K\end{array}$} & \multicolumn{1}{|c|}{$1 / 12$} & & $2 / 12$ & & $3 / 12$ & & $6 / 12$ & & 1 & & 2 \\
\hline \hline 39.6531 & 0.925 & 29.34 & - & 0.39 & 0.21 & 0.71 & 0.02 & 0.98 & 0.01 & 0.00 & 0.01 & 0.00 & 0.00 \\
38.6741 & 0.950 & 5.65 & 0.50 & 0.50 & 0.45 & 0.16 & 0.05 & 0.38 & 0.01 & 0.00 & 0.00 & 0.00 & 0.00 \\
37.7192 & 0.975 & 0.02 & 0.03 & 0.16 & 0.07 & 1.03 & 0.03 & 0.11 & 0.07 & 0.00 & 0.00 & 0.00 & 0.00 \\
36.7879 & 1.000 & 2.28 & 0.41 & 0.51 & 0.03 & 0.10 & 0.17 & 0.02 & 0.00 & 0.01 & 0.00 & 0.00 & 0.00 \\
30.1194 & 1.200 & 0.58 & 0.60 & 0.18 & 0.21 & 0.28 & 0.07 & 0.03 & 0.00 & 0.00 & 0.00 & 0.00 & 0.00 \\
22.3130 & 1.500 & 0.21 & 0.16 & 0.08 & 0.05 & 0.03 & 0.02 & 0.02 & 0.00 & 0.02 & 0.00 & 0.00 & 0.00 \\
\hline
\end{tabular}

Table 3

Values of $\mathcal{N}_{0.01}$, respectively, $\mathcal{N}_{0.001}$, given on the left, respectively, right, side, of the columns for various $K$ and $T$ corresponding to the option price $100 \cdot \operatorname{Put}(0.05, K, 2, T)$ in the model with mechanisms (4.3) and parameter values (5.3); at-the-money-strike: $100 \mathrm{~K}=94.1275$; zero-strike: $100 \mathrm{~K}=92.2259$. The spectral approximations $\mathcal{S}_{N}, N=1,2, \ldots$, are computed by the spectral-Laplace method.

\begin{tabular}{|c||cc|cc|cc|cc|c|c|c|c|}
\hline \multicolumn{3}{|c||}{$T$} & $1 / 12$ & & $2 / 12$ & & $3 / 12$ & $6 / 12$ & 1 & 2 & \\
$100 K$ & & & & & & & & & & & \\
\hline \hline 93 & 20 & 25 & 15 & 20 & 12 & 16 & 8 & 11 & 6 & 7 & 5 & 5 \\
94 & 20 & 24 & 15 & 20 & 12 & 16 & 8 & 11 & 6 & 7 & 5 & 5 \\
95 & 19 & 24 & 15 & 19 & 12 & 16 & 8 & 10 & 6 & 7 & 5 & 5 \\
96 & 18 & 23 & 14 & 19 & 12 & 15 & 8 & 10 & 6 & 7 & 5 & 5 \\
100 & 16 & 19 & 13 & 16 & 11 & 14 & 8 & 10 & 6 & 7 & 5 & 5 \\
110 & 10 & 20 & 10 & 15 & 9 & 11 & 7 & 9 & 6 & 7 & 5 & 5 \\
\hline
\end{tabular}

Table 4

This table displays the relative spectral errors $\left|\frac{\mathcal{S}_{\mathcal{N}_{\epsilon}}-\mathcal{S}_{\infty}}{\mathcal{S}_{\infty}}\right| \times 100$ (written as a percentage and rounded off to 2 decimal places) for various $K$ and $T$ associated with the option price $100 \cdot \operatorname{Call}(0.05, K, 2, T)$ for two values of $\epsilon$, namely, $\epsilon=0.01=1 \%$ and $\epsilon=0.001=0.1 \%$, given on the left, respectively, right, side, of the columns, in the model with mechanisms (4.3) and parameter values (5.3). The values of $\mathcal{N}_{0.01}$ and $\mathcal{N}_{0.001}$ are displayed in Table 3. Here $\mathcal{S}_{\mathcal{N}_{\epsilon}}$, respectively, $\mathcal{S}_{\infty}$, is computed by the spectral-Laplace method, respectively, Laplace method.

\begin{tabular}{|c||cc|cc|cc|cc|cc|cc|c|}
\hline & $T$ & $1 / 12$ & & $2 / 12$ & & $3 / 12$ & & $6 / 12$ & & 1 & & 2 \\
$100 K$ & & & & & & & & & & & & \\
\hline \hline 93 & 0.22 & 0.46 & 0.28 & 0.01 & 0.18 & 0.01 & 0.04 & 0.00 & 0.00 & 0.00 & 0.00 & 0.00 \\
94 & 0.09 & 0.28 & 0.22 & 0.01 & 0.15 & 0.01 & 0.04 & 0.00 & 0.00 & 0.00 & 0.00 & 0.00 \\
95 & 0.11 & 0.14 & 0.17 & 0.01 & 0.12 & 0.01 & 0.03 & 0.00 & 0.00 & 0.00 & 0.00 & 0.00 \\
96 & 0.26 & 0.05 & 0.23 & 0.01 & 0.10 & 0.01 & 0.03 & 0.00 & 0.00 & 0.00 & 0.00 & 0.00 \\
100 & 0.13 & 0.03 & 0.11 & 0.01 & 0.07 & 0.01 & 0.01 & 0.00 & 0.00 & 0.00 & 0.00 & 0.00 \\
110 & 1.48 & 0.02 & 0.24 & 0.02 & 0.05 & 0.03 & 0.00 & 0.00 & 0.00 & 0.00 & 0.00 & 0.00 \\
\hline
\end{tabular}

lead to catastrophic cancellation in the sense that a small relative roundoff error in evaluating one of the individual terms might dominate the value of the total sum. The smaller the $T$, the more likely this will occur since then the individual terms will tend to 0 more slowly as $n \rightarrow \infty$. This problem could be to some extent remedied by working with a higher system precision when $T$ is small. On a related note, during the testing of the explicit-spectral method in the

Copyright (C) by SIAM. Unauthorized reproduction of this article is prohibited. 
Table 5

Option prices $100 \cdot \mathrm{Call}(0.05, K, 2, T)$ for various $K$ and $T$, rounded off to four decimals, computed by the Laplace method in the model with mechanisms (4.1) and parameter values (5.2).

\begin{tabular}{|cc|cccccc|}
\hline & $T$ & $1 / 12$ & $2 / 12$ & $3 / 12$ & $6 / 12$ & 1 & 2 \\
$100 K$ & $-\log K$ & & & & & & \\
\hline \hline 39.6531 & 0.925 & 0.0012 & 0.0032 & 0.0028 & 0.0010 & 0.0002 & 0.0000 \\
38.6741 & 0.950 & 0.0791 & 0.0642 & 0.0443 & 0.0146 & 0.0029 & 0.0005 \\
37.7192 & 0.975 & 0.3704 & 0.2435 & 0.1620 & 0.0557 & 0.0123 & 0.0022 \\
36.7879 & 1.000 & 0.8478 & 0.5434 & 0.3646 & 0.1329 & 0.0320 & 0.0059 \\
30.1194 & 1.200 & 6.3687 & 4.9819 & 3.9288 & 2.0588 & 0.7433 & 0.1855 \\
22.3130 & 1.500 & 13.9053 & 12.0885 & 10.5039 & 6.9582 & 3.3352 & 1.0441 \\
\hline
\end{tabular}

Table 6

Option prices $100 \cdot \operatorname{Put}(0.05, K, 2, T)$ for various $K$ and $T$ computed by the Laplace method in the model with mechanisms (4.3) and parameter values (5.3).

\begin{tabular}{|c|c|c|c|c|c|c|}
\hline $100 K{ }^{T}$ & $1 / 12$ & $2 / 12$ & $3 / 12$ & $6 / 12$ & 1 & 2 \\
\hline 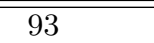 & 0.0143 & 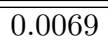 & 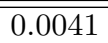 & $\overline{0.0016}$ & 0.0005 & 0.0001 \\
\hline 94 & 0.1050 & 0.0518 & 0.0316 & 0.0123 & 0.0041 & 0.0009 \\
\hline 95 & 0.2959 & 0.1500 & 0.0927 & 0.0366 & 0.0124 & 0.0028 \\
\hline 96 & 0.5897 & 0.3071 & 0.1925 & 0.0774 & 0.0265 & 0.0060 \\
\hline 100 & 2.6465 & 1.5254 & 1.0074 & 0.4313 & 0.1525 & 0.0351 \\
\hline 110 & 11.0049 & 7.6833 & 5.6312 & 2.7631 & 1.0533 & 0.2489 \\
\hline
\end{tabular}

model with mechanisms (4.1) and parameters (5.2), we found that it is very hard to compute accurately the co-eigendensities $\nu_{n}(y)$ given by (4.2) for large $n$. As an example, for the same parameter values as in the numerical experiment, large errors in the evaluation of $\nu_{35}(y)$ with $\mathrm{R}$ occur for $y>1.5$. This is because (4.2) consists of a sum where the terms alternate in sign and so catastrophic cancellation can occur. Although this issue did not form a problem for computing the numbers in Table 1 due to the smoothing effect of the payoff kernel, it is something to keep in mind when applying the explicit-spectral method. On the other hand, we did not find such problems when computing the co-eigendensities by the spectral-Laplace method (in combination with Filon's method for the Laplace inversion).

5.2. Computation times. We now perform an experiment where we look at computation times of the various methods. In order to be able to compare the computation times of all the methods, we consider again bond call option prices Call $(0.05, K, 2, T)$ in the model with mechanisms (4.1) and parameter values (5.2). In particular we provide for various $\bar{N}$ the computation times of $\mathcal{S}_{N}(K, T)$ simultaneously for all $K \in \mathbb{K}, T \in \mathbb{T}$ and $N \in\{1, \ldots, \bar{N}\}$, where

$$
\mathbb{K}=\{\exp (-0.91875-0.00625 \cdot j): j=1,2, \ldots, 96\}, \quad \mathbb{T}=\left\{\frac{1}{12}, \frac{2}{12}, \frac{3}{12}, \frac{6}{12}, 1,2\right\} .
$$

We do this exercise for three different methods, namely, the explicit-spectral method, the spectral-Laplace method where we use the explicit expressions from section 4.1 to evaluate the functions $A(\lambda)$ and $\mathrm{e}^{-F(\lambda)}$, and a second version of the latter method, referred to as 
the numerical spectral-Laplace method, where we compute the two functions by numerically solving the (system of) first order homogeneous linear ODEs,

$$
\left\{\begin{array}{ll}
\frac{\mathrm{d}}{\mathrm{d} \lambda} A^{\prime}(\lambda)=-A^{\prime}(\lambda) \frac{\psi^{\prime}(\lambda)-\psi^{\prime}(\theta)}{\psi(\lambda)}, & A^{\prime}(\theta)=1, \\
\frac{\mathrm{d}}{\mathrm{d} \lambda} A(\lambda)=A^{\prime}(\lambda), & A(\theta)=0,
\end{array} \quad \frac{\mathrm{d}}{\mathrm{d} \lambda} \mathrm{e}^{-F(\lambda)}=-\mathrm{e}^{-F(\lambda)} \frac{\phi(\lambda)-\phi(\theta)}{\psi(\lambda)}, \quad \mathrm{e}^{-F(\theta)}=1 ;\right.
$$

see (2.14) and (2.12). Note that we have set $A^{\prime}(\theta)=1$ instead of (3.1), which we can do as long as we also use $A^{\prime}(\theta)=1$ in the computation of the eigenfunctions in Proposition 3.1. This avoids having to numerically compute the (improper) integral (3.1). Further we provide for various sets $\overline{\mathbb{T}}$ the computation times of $\mathcal{S}_{\infty}(K, T)$ simultaneously for all $K \in \mathbb{K}$ and $T \in \overline{\mathbb{T}}$ by using the Laplace method where the CBI exponents $\Psi_{T}(\lambda)$ and $\Phi_{T}(\lambda)$ are evaluated by using the explicit formulas from section 4.1 and what we call the numerical Laplace method, where we solve numerically the ODEs (2.5) and (2.6) in order to evaluate these CBI exponents. The reason for considering both the numerical spectral-Laplace and the Laplace method is to have an idea about computation times in the case where explicit expressions for $A, F, \Psi_{T}$, and $\Phi_{T}$ are not available.

The Laplace inversion is done in the same way as in section 5.1 and with the same parameter choices; in particular the number of discretization points we work with is $2^{14}$. For solving numerically all the ODEs involved we use the zvode() function in the deSolve R-package [36] which can deal with complex-valued functions. Here we used a value of $10^{-8}$ for the absolute and relative tolerance levels in zvode() instead of the default value of $10^{-6}$, which results in a relative error (i.e., the one introduced by numerically solving ODEs) for $\mathcal{S}_{N}(K, T)$ or $\mathcal{S}_{\infty}(K, T)$ of well below $1 \%$ for pretty much all the considered values of $K, T$, and $N$.

The computation times are displayed in Table 7 and were produced by using an Intel Core i5-4590 CPU @ 3.30GHz $\times 4$ and 7.7 GB memory. They confirm the general remarks made in section 3.3. The time it takes to do a numerical Laplace inversion consists largely of evaluations of the Laplace transform. For the Laplace method this consists of evaluating $A, \bar{A}$ and twice $F$ (see (2.15) and (2.16)), whereas for the spectral-Laplace method it only involves $A$ and $F$. This explains why the spectral-Laplace method is in our example faster (even for $\bar{N}=50$ ) than the Laplace method even if the latter is applied to one maturity time only. Although more Laplace inversions need to be done with the spectral-Laplace method as $N$ in $\mathcal{S}_{N}$ increases, due to the special product structure in $n$ of the Laplace transform of the

Table 7

The left-hand side of the table gives the computation times in seconds for computing with the three spectral methods, $\mathcal{S}_{N}(K, T)$ simultaneously for all $K \in \mathbb{K}$, for all $T \in \mathbb{T}$, and for all $N \in\{1, \ldots, \bar{N}\}$, where $\bar{N}$ varies. The right-hand side of the table gives, for various $\overline{\mathbb{T}}$, the computation times of $\mathcal{S}_{\infty}(K, T)$ simultaneously for all $K \in \mathbb{K}$ and $T \in \overline{\mathbb{T}}$ with the Laplace and the numerical Laplace method. Here $\mathbb{K}$ and $\mathbb{T}$ are given by (5.4) and $\mathcal{S}_{N}(K, T)$ and $\mathcal{S}_{\infty}(K, T)$ correspond to the option price $100 \cdot \operatorname{Call}(0.05, K, 2, T)$ in the model with mechanisms (4.1) and parameter values (5.2).

\begin{tabular}{|c|c|c|c|c|c|c|c|c|}
\hline & \multicolumn{3}{|c|}{$\bar{N}$} & \multirow[b]{2}{*}{ Method } & \multicolumn{4}{|c|}{$\overline{\mathbb{T}}$} \\
\hline Method & 5 & 20 & 50 & & $\left\{\frac{1}{12}\right\}$ & $\{2\}$ & $\left\{\frac{1}{12}, \frac{2}{12}, \frac{3}{12}\right\}$ & $\mathbb{T}$ \\
\hline Spectral-explicit & 0.04 & 0.1 & 0.4 & & & & & \\
\hline Spectral-Laplace & 0.1 & 0.1 & 0.3 & Laplace & 0.6 & 0.6 & 1.7 & 3.3 \\
\hline Numerical spectral-Laplace & 0.6 & 0.6 & 0.8 & Numerical Laplace & 17 & 42 & 60 & 167 \\
\hline
\end{tabular}

Copyright (C) by SIAM. Unauthorized reproduction of this article is prohibited. 
co-eigenmeasure $\mathcal{V}_{n}$, we do not need more evaluations of the functions $A$ and $F$ as $N$ increases, whereas for the spectral-explicit method more and more incomplete gamma functions need to be evaluated. This explains why the computation time of the spectral-explicit method grows faster than with (both) the spectral-Laplace method(s) as the number of terms in the spectral expansion increases from 5 to 50 . As noted before, one of the main advantages of the spectral methods is that it is very cheap to perform them for multiple expiry times (assuming the number of terms in the spectral expansion stays constant). In contrast we see from Table 7 that for the Laplace method the computation time increases almost linearly with the number of expiry times, which is because in this case the Laplace transform (i.e., the CBI exponents $\Psi_{T}(\lambda)$ and $\left.\Phi_{T}(\lambda)\right)$ needs to be reevaluated whenever $T$ changes. When we look at the numerical versions, the speed advantage of the spectral-Laplace over the Laplace method becomes even bigger. This is because for the numerical spectral-Laplace method we solve the ODE system (5.5) only once on a $\lambda$-grid consisting of $2^{14}$ points, whereas for the numerical Laplace method we solve the ODE system (2.5)-(2.6) $2^{14}$ times on a $T$-grid given by $\overline{\mathbb{T}}$, namely, once for each of the $2^{14}$ values of $\lambda$ that we need for the Laplace inversion. Also note that, as can be seen from Table 7 when looking at the $\overline{\mathbb{T}}=\left\{\frac{1}{12}\right\}$ and $\overline{\mathbb{T}}=\{2\}$ entries, the numerical Laplace method gets more expensive as the value of the maturity time increases (in contrast to the nonnumerical Laplace method), which is because the ODEs then have to be solved on a longer time period as the initial condition is at $T=0$. In that sense the numerical Laplace method and (numerical) spectral method complement each other, since the former is weak for large $T$ where the latter shines, whereas it is less slow for small $T$ where the spectral methods might not be applicable (if $T \leq \underline{T}$ ) or might suffer from catastrophic cancellation (when $T$ is close to $\underline{T}$ ).

We remark that, as is typical when providing computation times, Table 7 can only provide a snapshot of the speed differences of the methods. Although the absolute values of these speed differences can certainly change when, e.g., different methods are chosen for the numerical Laplace inversion and ODE solving, the overall picture as described in the above paragraph should still hold.

6. Conclusion. We have shown, under a mild condition on the branching mechanism, that the pricing semigroup of a positive affine short rate process admits a discrete spectral representation. The spectral expansion is obtained in terms of eigenfunctions and co-eigenmeasures that are derived directly from the branching and immigration mechanisms of the short rate process. Thus, the spectral approach does not assume the Laplace transform of the pricing semigroup to be explicitly given. In that sense, we have gained a significant analytical simplification for the pricing of European interest rate derivatives in one-dimensional affine term structure models. Moreover, the theoretical and numerical results show that the two methods, based on the spectral expansion, that we suggested, can compute option prices with great accuracy and have a significant speed advantage compared to the Laplace method, especially when computing option prices for multiple expiry times or when the underlying ODEs have to be solved numerically.

\section{Appendix A. Proofs.}

A.1. Some lemmas. We start with several lemmas containing statements that either have been used in the main body of the paper or will be used later on for the proof of the main 
results. The first lemma provides some properties of the functions $A$ and $F$ defined in (2.11) and (2.12).

Lemma A.1. The function $A$, respectively, $F$, is analytic on (i.e., can be analytically extended to) $\left\{z \in \mathbb{C}: \mathfrak{R e}(z)>-\left(R_{\psi} \wedge \underline{\theta}\right)\right\}$, respectively, $\left\{z \in \mathbb{C}: \mathfrak{R e}(z)>-\left(R_{\psi} \wedge R_{\phi} \wedge \underline{\theta}\right)\right\}$. If $R_{\psi}=0$, respectively, $R_{\psi} \wedge R_{\phi}=0$, then $A$, respectively, $F$, can (still) be continuously extended to $\mathbb{R}_{\geq 0}^{+}$. Hence $R_{A}=R_{\psi} \wedge \underline{\theta}$ and $R_{F}=R_{\psi} \wedge R_{\phi} \wedge \underline{\theta}$. For $\lambda \in\left(-R_{A}, \infty\right) \cup\{0\}$, respectively, $\lambda \in\left(-R_{F}, \infty\right) \cup\{0\}$, we have that $A$, respectively, $F$, admits the expression (2.14), respectively, (2.12). Finally the functions $\nu$ and $\omega$ are well-defined (up to a set of 0 Lebesgue measure) via (2.22) and (2.23).

Proof. The function $A$ as defined in (2.11) satisfies the differential equation $\psi(\lambda) A^{\prime}(\lambda)=$ $\psi^{\prime}(\theta) A(\lambda)$ for $\lambda>\theta$. Differentiating on both sides and rearranging gives $A^{\prime \prime}(\lambda)=-\frac{\psi^{\prime}(\lambda)-\psi^{\prime}(\theta)}{\psi(\lambda)}$ $A^{\prime}(\lambda)$, which leads to

$$
A^{\prime}(\lambda)=A^{\prime}(\theta) \exp \left(-\int_{\theta}^{\lambda} \frac{\psi^{\prime}(u)-\psi^{\prime}(\theta)}{\psi(u)} d u\right), \quad \lambda \geq \theta .
$$

By (2.11) and (2.13), $A(\theta)=0$ and $A(\infty)=1$ and so integrating both sides of the above equation with respect to $\lambda$ from $\theta$ to $\infty$ gives $A^{\prime}(\theta)$, which then shows that (2.14) holds for $\lambda \geq \theta$. Since $\psi^{\prime}(\theta)>0$ and $\psi(0)=-1$ and by definition of $R_{\psi}, R_{\phi}$, and $\underline{\theta}$, the right-hand side of (2.14), respectively, (2.12), is well-defined for $\lambda>-\left(R_{\psi} \wedge \underline{\theta}\right)$ and $\lambda=0$, respectively, $\lambda>-\left(R_{\psi} \wedge R_{\phi} \wedge \underline{\theta}\right)$ and $\lambda=0$. Hence $A$ and $F$ can be continuously extended to those respective regions via (2.14) and (2.12).

Set $\psi_{\theta}(\lambda)=\psi(\lambda+\theta)$ and $\phi_{\theta}(\lambda)=\phi(\lambda+\theta)-\phi(\theta)$. Then the pair $\left(\psi_{\theta}, \phi_{\theta}\right)$ satisfies the conditions of [4, Proposition 3.1] and so by [4, Proposition 3.1(2)] there exists a nonnegative integrable function $\nu_{\theta}$ on $\mathbb{R}^{+}$such that $\int_{0}^{\infty} e^{-\lambda y} \nu_{\theta}(y) d y=e^{-F(\lambda+\theta)}-e^{-F(\infty)}$ for $\lambda \geq 0$. Similarly, by [4, Corollary 3.4] there exists a probability density function $\omega_{\theta}$ on $\mathbb{R}^{+}$such that $\int_{0}^{\infty} e^{-\lambda y} \omega_{\theta}(y) d y=1-A(\lambda+\theta)$ for $\lambda \geq 0$. So setting $\omega(y)=e^{\theta y} \omega_{\theta}(y)$ and $\nu(y)=e^{\theta y} \nu_{\theta}(y)$, we have that (2.22) and (2.23) hold for $\lambda \geq \theta$. We have already shown that $A$ and $F$ can be extended to larger domains. Therefore by standard properties of the Laplace transform it follows that $A(z)$, respectively, $F(z)$, is analytic on $\mathfrak{R e}(z)>-\left(R_{\psi} \wedge \underline{\theta}\right)$, respectively, $\mathfrak{R e}(z)>-\left(R_{\psi} \wedge R_{\phi} \wedge \underline{\theta}\right)$. Hence $R_{A}=R_{\psi} \wedge \underline{\theta}$ and $R_{F}=R_{\psi} \wedge R_{\phi} \wedge \underline{\theta}$ and (2.22), respectively, (2.23), hold for $\lambda \in\left(-R_{A}, \infty\right) \cup\{0\}$, respectively, $\lambda \in\left(-R_{F}, \infty\right) \cup\{0\}$.

The second lemma shows the connection between the CBI exponents $\Psi_{t}(\lambda)$ and $\Phi_{t}(\lambda)$ and the functions $A$ and $F$.

Lemma A.2. For all $\lambda>-R_{A}$, the $O D E(2.3)$ admits a unique solution $\left(\Psi_{t}(\lambda)\right)_{t \geq 0}$. Set $\Phi_{t}(\lambda)=\int_{0}^{t} \phi\left(\Psi_{s}(\lambda)\right) d s$ and if $R_{A}=0$, set $\Psi_{t}(0)=\lim _{\lambda \downarrow 0} \Psi_{t}(\lambda)$. Then (2.15) and (2.16) hold for all $t \geq 0$. In addition (2.2) holds for all $t, x \geq 0$ and $\lambda \in\left(-R_{F}, \infty\right) \cup\{0\}$.

Proof. Since $A$ satisfies the differential equation $\psi(\lambda) A^{\prime}(\lambda)=\psi^{\prime}(\theta) A(\lambda)$ for $\lambda>-R_{A}$ (see the proof of Lemma A.1), one can easily show that the right-hand side of (2.15) solves the ODE (2.3) for all $t \geq 0$ and $\lambda>-R_{A}$. Since $A$ is an increasing function, it follows that for any $\lambda>-R_{A}$, the right-hand side of (2.15) stays in the interval $\left(-R_{A}, \infty\right)$ for all $t \geq 0$. Since $\psi$ is certainly locally Lipschitz on $\left(-R_{A}, \infty\right)$, it follows by standard ODE theory that 
the right-hand side of (2.15) is the unique solution to the ODE (2.3). Hence (2.15) must hold for $\lambda>-R_{A}$. Using (2.12) (which by Lemma A.1 holds for $\lambda>-R_{F}$ ) and (2.3), it is easy to show that (2.16) holds for $\lambda>-R_{F}$. Then by [20, Theorem 2.14(b)], (2.2) holds for all $t, x \geq 0$ and $\lambda>-R_{F}$. Finally, by taking limits as $\lambda \downarrow 0$ one shows that if $R_{A}=0$, then (2.15) holds for $\lambda=0$ as well, and if $R_{F}=0$, then (2.16) and (2.2) also hold for $\lambda=0$.

The next lemma provides the Laplace transform of the signed measures $\mathcal{V}_{n}$, which we recall is defined by $\mathcal{V}_{n}(d y)=e^{-F(\infty)} \delta_{0}(d y)+\nu_{n}(y) d y$ for $n \geq 0$. Further, it gives an upper bound for the Laplace transform of the total variation measure of $\mathcal{V}_{n}$ denoted by $\left|\mathcal{V}_{n}\right|$.

Lemma A.3. For all $n \geq 0$ and for all $\lambda \in\left(-R_{F}, \infty\right) \cup\{0\}$,

$$
\begin{gathered}
\int_{0}^{\infty} e^{-\lambda y} \mathcal{V}_{n}(d y)=A(\lambda)^{n} e^{-F(\lambda)}, \\
\int_{0}^{\infty} e^{-\lambda y}\left|\mathcal{V}_{n}\right|(d y) \leq(2-A(\lambda))^{n} e^{-F(\lambda)} .
\end{gathered}
$$

Proof. Equation (A.1) follows directly from the definitions (2.22)-(2.25) and (2.27), the binomial theorem, and the well-known relation regarding Laplace transforms and convolutions; see the proof of (5.7) in [4] for more details. By the same arguments and using the bound $\left|\mathcal{W}_{n}(y)\right| \leq \sum_{j=1}^{n}\left(\begin{array}{c}n \\ j\end{array}\right) \omega^{* j}(y)$, the bound (A.2) follows.

We precede the proof with the following useful bounds respectively on the Sheffer polynomials $\left(\mathcal{L}_{n}\right)_{n \geq 0}$ and their derivatives; see (2.18) for their definition.

Lemma A.4. Let $k \geq 0$ and $R \in\left(0, R_{G}\right)$. Then there exists $C=C(R, k)>0, M=$ $M(R)>0$, such that for any $x \geq 0, n \geq 0$,

$$
\left|\frac{\partial^{k}}{\partial x^{k}} \mathcal{L}_{n}(x)\right| \leq C \frac{e^{M x}}{R^{n+1}},
$$

and we have

$$
\frac{\partial^{k}}{\partial x^{k}} G_{x}(z)=\sum_{n=k}^{\infty} \frac{\partial^{k}}{\partial x^{k}} \mathcal{L}_{n}(x) z^{n}, \quad|z|<R_{G},
$$

where the series converges locally uniformly in $x$.

Proof. First observe that the mapping $z \mapsto \frac{\partial^{k}}{\partial x^{k}} G_{x}(z)=(-1)^{k}(\bar{A}(z)-\theta)^{k} G_{x}(z)$ is analytic at 0 and the radius of convergence of its Taylor series at 0 is $R_{G}$. Moreover it is clear that $(x, z) \mapsto G_{x}(z) \in C^{\infty}\left([0, \infty) \times\left(-R_{G}, R_{G}\right)\right)$ so that, for all $x \geq 0$,

$$
\frac{1}{n !} \frac{\partial^{n}}{\partial z^{n}}\left(\frac{\partial^{k}}{\partial x^{k}} G_{x}(z)\right)_{z=0}=\frac{\partial^{k}}{\partial x^{k}}\left(\frac{1}{n !}\left(\frac{\partial^{n}}{\partial z^{n}} G_{x}(z)\right)_{z=0}\right)=\frac{\partial^{k}}{\partial x^{k}} \mathcal{L}_{n}(x)
$$

and then, for any $R \in\left(0, R_{G}\right)$, an application of the Cauchy's formula yields that

$$
\frac{\partial^{k}}{\partial x^{k}} \mathcal{L}_{n}(x)=\frac{1}{2 \pi i} \oint \frac{(-1)^{k}(\bar{A}(z)-\theta)^{k} G_{x}(z)}{z^{n+1}} d z
$$

Copyright (C) by SIAM. Unauthorized reproduction of this article is prohibited. 
where the contour is a circle centered at 0 and of radius $R$. Next, since clearly $\bar{A}$ and $F \circ \bar{A}$ are analytic at 0 with $\min \left(R_{\bar{A}}, R_{F \circ \bar{A}}\right) \geq R_{G}>R$, we get that $\sup _{|z|=R}|\bar{A}(z)|, \sup _{|z|=R}|F(\bar{A}(z))| \in$ $(0, \infty)$ and hence that there exist $C(R, k)>0$ and $M(R)=M>0$ such that

$$
\left|\frac{\partial^{k}}{\partial x^{k}} \mathcal{L}_{n}(x)\right| \leq \frac{1}{2 \pi} \oint\left|\frac{(-1)^{k}(\bar{A}(z)-\theta)^{k} e^{F(\bar{A}(z))} e^{-x(\bar{A}(z)-\theta)}}{z^{n+1}}\right| d z \leq C \frac{e^{M x}}{R^{n+1}} .
$$

Note that since $\bar{A}(0)=\theta$, one may choose $R$ such that $M(R)=\sup _{|z|=R}|\bar{A}(z)-\theta|<R_{G}$. This proves (A.3) and shows that for any $z$ satisfying $|z|<R_{G}$, there exist $R \in\left(|z|, R_{G}\right)$, $C(R, k), M(R)>0$ such that

$$
\sum_{n=k}^{\infty}\left|\frac{\partial^{k}}{\partial x^{k}} \mathcal{L}_{n}(x) z^{n}\right| \leq \frac{C}{R} e^{M x} \sum_{n=k}^{\infty}\left(\frac{|z|}{R}\right)^{n}<\infty,
$$

which proves (A.4).

A.2. Proof of Theorem 2.2. We split the proof into several steps. We first show that for $T>\underline{\mathrm{T}}$ and $\lambda>-\bar{\lambda}_{T}$ the pricing semigroup coincide on $\mathcal{P}_{\lambda}$ with the following two operators, defined on $\mathcal{B}\left(\mathbb{R}_{\geq 0}^{+}\right)$, by

$$
S_{T} f(x)=\sum_{n=0}^{\infty} e^{-\lambda_{n} T} \mathcal{L}_{n}^{\theta}(x) \mathcal{V}_{n} f \quad \text { and } \quad \overline{S_{T}} f(x)=\int_{0}^{\infty} f(y) \sum_{n=0}^{\infty} e^{-\lambda_{n} T} \mathcal{L}_{n}^{\theta}(x) \mathcal{V}_{n}(d y)
$$

Lemma A.5. For all $T>\underline{\mathrm{T}}, x \geq 0$, and $f \in \mathcal{P}_{\lambda}$ with $\lambda>-\bar{\lambda}_{T}$, we have $\overline{S_{T}} f(x)=S_{T} f(x)$.

Proof. By definition (2.21), there exists $C>0$ such that $|f(x)| \leq C e^{-\lambda x}$ for almost every $x \geq 0$. Thus, recalling (2.26),

$$
\begin{aligned}
\sum_{n=0}^{\infty} e^{-\lambda_{n} T}\left|\mathcal{L}_{n}^{\theta}(x)\right|\left|\mathcal{V}_{n}\right||f| & \leq C e^{-\phi(\theta) T} \sum_{n=0}^{\infty}\left|\mathcal{L}_{n}(x)\right| e^{-\psi^{\prime}(\theta) n T}\left|\mathcal{V}_{n}\right| e_{\lambda} \\
& \leq C e^{-\phi(\theta) T} e^{-F(\lambda)} \sum_{n=0}^{\infty}\left|\mathcal{L}_{n}(x)\right|\left(e^{-\psi^{\prime}(\theta) T}(2-A(\lambda))\right)^{n}
\end{aligned}
$$

where the last inequality follows from (A.2). One can check that $\lambda>-\bar{\lambda}_{T}$ is equivalent to $\lambda>-R_{F}$ and $2-A(\lambda)<e^{\psi^{\prime}(\theta)(T-\underline{T})}$. Since moreover $e^{-\psi^{\prime}(\theta) \underline{T}}=R_{G}$, by definition (see (2.19)), we conclude by (2.18),

$$
\sum_{n=0}^{\infty} e^{-\lambda_{n} T}\left|\mathcal{L}_{n}^{\theta}(x)\right|\left|\mathcal{V}_{n}\right||f|<\infty
$$

By the Fubini theorem, this shows that $\overline{S_{T}} f$ is well-defined and satisfies $\overline{S_{T}} f=S_{T} f$.

Next, we show that the operators $S_{T}$ and $P_{T}$ coincide on a subset of $\left\{e_{\lambda}: \lambda \in\left(-R_{F}, \infty\right) \cup\right.$ $\{0\}\}$.

Lemma A.6. For all $x \geq 0, T \geq 0$ and $\lambda \in\left(-R_{F}, \infty\right) \cup\{0\}$ such that $|A(\lambda)|<e^{\psi^{\prime}(\theta)(T-\underline{\mathrm{T}})}$, we have $S_{T} e_{\lambda}(x)=P_{T} e_{\lambda}(x)$. 
Proof. Let $x, T \geq 0$ and $\lambda \in\left(-R_{F}, \infty\right) \cup\{0\}$. Then by (2.2) (see also Lemma A.2), (2.15), (2.16), and (2.17),

$$
\begin{aligned}
P_{T} e_{\lambda}(x) & =e^{-\phi(\theta) T} e^{F\left(\bar{A}\left(e^{-\psi^{\prime}(\theta) t} A(\lambda)\right)\right)} e^{-F(\lambda)} e^{-x \bar{A}\left(e^{-\psi^{\prime}(\theta) t} A(\lambda)\right)} \\
& =e^{-\theta x} e^{-\phi(\theta) T} e^{-F(\lambda)} G_{x}\left(e^{-\psi^{\prime}(\theta) T} A(\lambda)\right)
\end{aligned}
$$

On the other hand, assume in addition that $|A(\lambda)|<e^{\psi^{\prime}(\theta)(T-\underline{T})}$. Then by (A.1), (2.26), (2.19), and (2.18),

$$
\begin{aligned}
S_{T} e_{\lambda}(x) & =\sum_{n=0}^{\infty} e^{-\lambda_{n} T} \mathcal{L}_{n}^{\theta}(x) A(\lambda)^{n} e^{-F(\lambda)} \\
& =e^{-\theta x} e^{-\phi(\theta) T} e^{-F(\lambda)} \sum_{n=0}^{\infty} \mathcal{L}_{n}(x)\left(e^{-\psi^{\prime}(\theta) T} A(\lambda)\right)^{n} \\
& =e^{-\theta x} e^{-\phi(\theta) T} e^{-F(\lambda)} G_{x}\left(e^{-\psi^{\prime}(\theta) T} A(\lambda)\right) .
\end{aligned}
$$

Hence the lemma is proved.

We are now ready to prove Theorem 2.2. Let $T>\underline{\mathrm{T}}$ and $f \in \mathcal{P}_{\lambda}$ for some $\lambda>-\bar{\lambda}_{T}$. Then, from Lemma A.5, Lemma A.6, and uniqueness of the Laplace transform we have gained that $P_{t} f=\overline{S_{t}} f=S_{t} f$, which proves Theorem 2.2(1). We now turn to the proof of Theorem 2.2(2). Let $p, k$ be some nonnegative integers. From the proof of Lemma A.5, it is easily seen that there exist $C>0$ and $R \in\left(0, R_{G}\right)$ such that for $n$ large enough, $\left|\left(-\lambda_{n}\right)^{p} e^{-\lambda_{n} T} \mathcal{V}_{n} f\right| \leq C R^{n}$. This combined with Leibniz's rule entails that

$$
\begin{aligned}
\sum_{n=0}^{\infty}\left|\left(-\lambda_{n}\right)^{p} e^{-\lambda_{n} T} \frac{\partial^{k}}{\partial x^{k}} \mathcal{L}_{n}^{\theta}(x) \mathcal{V}_{n} f\right| & \leq C \sum_{n=0}^{\infty}\left|\frac{\partial^{k}}{\partial x^{k}} \mathcal{L}_{n}^{\theta}(x)\right| R^{n} \\
& \leq C e^{-\theta x} \sum_{j=0}^{k}\left(\begin{array}{c}
k \\
j
\end{array}\right) \theta^{k-j} \sum_{n=0}^{\infty}\left|\frac{\partial^{j}}{\partial x^{j}} \mathcal{L}_{n}(x)\right| R^{n}
\end{aligned}
$$

where, from (A.4), the series on the right-hand side is locally uniformly convergent in $x$. So $\frac{\partial^{p}}{\partial T^{p}} \frac{\partial^{k}}{\partial x^{k}} \sum_{n=0}^{N} e^{-\lambda_{n} T} \frac{\partial^{k}}{\partial x^{k}} \mathcal{L}_{n}^{\theta}(x) \mathcal{V}_{n} f$ converges locally uniformly in $(T, x)$ as $N \rightarrow \infty$. Since we already showed that $P_{T} f(x)=S_{T} f(x)=\sum_{n=0}^{\infty} e^{-\lambda_{n} T} \mathcal{L}_{n}^{\theta}(x) \mathcal{V}_{n} f$, this proves, by a classical result in analysis (see, e.g., [35, Theorem 7.17]), the claim (2.29) and shows that $(T, x) \longmapsto$ $P_{T} f(x) \in C^{\infty^{2}}\left((\underline{\mathrm{T}}, \infty) \times \mathbb{R}_{\geq 0}^{+}\right)$. This completes the proof of Theorem 2.2(2).

Next, we prove Theorem 2.2(3). Let $T>\underline{\mathrm{T}}$ and $f \in \mathcal{P}_{\lambda}$ for some $\lambda>-\bar{\lambda}_{T}$. Let

$$
S(\beta)=\psi^{\prime}(\theta)(T-\underline{\mathrm{T}})-\ln (2-A(\beta)) .
$$

By the definition of $\bar{\lambda}_{T}$ in (2.20), we have $S\left(-\bar{\lambda}_{T}\right) \geq 0$. Since $A$ is an increasing function, it follows that $S(\cdot)$ is also an increasing function, and thus $S=S(\lambda)>0$ since $\lambda>-\bar{\lambda}_{T}$. Let $\varepsilon \in(0, S)$ and set $R=R(\varepsilon)=e^{-\psi^{\prime}(\theta) \underline{\mathrm{T}}-\varepsilon} \in\left(0, R_{G}\right)$. Then recalling that $\mathcal{L}_{n}^{\theta}(x)=$ $e^{-\theta x} \mathcal{L}_{n}(x)$, the representation (2.28), the Leibniz's formula, and (A.3) show that there exist $M=M(\varepsilon)>0$ such that for any integer $k \geq 0$ there exist $C(j, \varepsilon)>0, j=0, \ldots k$, such that $C_{\theta, \varepsilon, k}=\sum_{j=0}^{k}\left(\begin{array}{c}k \\ j\end{array}\right) \theta^{k-j} C(j, \varepsilon)>0$ and 


$$
\begin{aligned}
\left|\frac{\partial^{k}}{\partial x^{k}} P_{t} f(x)-\sum_{n=0}^{N-1} e^{-\lambda_{n} T} \frac{\partial^{k}}{\partial x^{k}} \mathcal{L}_{n}^{\theta}(x) \mathcal{V}_{n} f\right| & \leq \sum_{n=N}^{\infty} e^{-\lambda_{n} T}\left|\frac{\partial^{k}}{\partial x^{k}} \mathcal{L}_{n}^{\theta}(x)\right|\left|\mathcal{V}_{n} f\right| \\
& \leq C_{\theta, \varepsilon, k} e^{-(\theta-M) x-\phi(\theta) T} \sum_{n=N}^{\infty} \frac{e^{-n \psi^{\prime}(\theta) T}}{R^{n+1}}\left|\mathcal{V}_{n} f\right|
\end{aligned}
$$

where we have used (2.26) in the last inequality. Since $f \in \mathcal{P}_{\lambda}$, there exists $c_{f, \lambda}>0$ such that $\left|\mathcal{V}_{n} f\right| \leq c_{f, \lambda} e^{-F(\lambda)}(2-A(\lambda))^{n}$; see (A.2). Hence, recalling $R=e^{-\psi^{\prime}(\theta) \underline{T}-\varepsilon}$, we get, with $C_{1}=c_{f, \lambda} C_{\theta, \varepsilon, k}>0$,

$$
\left|\frac{\partial^{k}}{\partial x^{k}} P_{t} f(x)-\sum_{n=0}^{N-1} e^{-\lambda_{n} T} \frac{\partial^{k}}{\partial x^{k}} \mathcal{L}_{n}^{\theta}(x) \mathcal{V}_{n} f\right| \leq C_{1} e^{-(\theta-M) x-\phi(\theta) T-F(\lambda)+\psi^{\prime}(\theta) \underline{\mathrm{T}}+\varepsilon} \sum_{n=N}^{\infty} e^{-n(S-\varepsilon)} .
$$

As $\varepsilon$ was chosen such that $S-\varepsilon>0$, Theorem 2.2(3) follows with $C=C(k, \varepsilon, \lambda, f)=$ $c_{f, \lambda} C_{\theta, \varepsilon, k} e^{-F(\lambda)+\psi^{\prime}(\theta) \underline{\mathbf{T}}+\varepsilon}$.

A.3. Proof of Theorem 2.3. Let $T \geq 0$ and $\lambda \in\left(-R_{F}, \infty\right) \cup\{0\}$ be such that $|A(\lambda)|<$ $e^{\psi^{\prime}(\theta)(T-\underline{\mathrm{T}})}$. Then (2.28) holds for $f=e_{\lambda}$ by Lemma A.6. Using the equality (A.1) instead of the inequality (A.2), the rest of the theorem follows along the same lines as Theorem 2.2.

A.4. Proof of Proposition 3.1. The first claim follows from Lemma 2.2 in [29]. Then, since, for any $n \geq 0, \mathcal{L}_{n}^{\theta} \in C_{0}\left(\mathbb{R}_{\geq 0}^{+}\right)$, we get that

$$
\lim _{t \downarrow 0} \frac{P_{t} \mathcal{L}_{n}^{\theta}(x)-\mathcal{L}_{n}^{\theta}(x)}{t}=\lim _{t \downarrow 0} \frac{e^{-\lambda_{n} t}-1}{t} \mathcal{L}_{n}^{\theta}(x)=-\lambda_{n} \mathcal{L}_{n}^{\theta}(x) \in C_{0}\left(\mathbb{R}_{\geq 0}^{+}\right),
$$

from where we readily complete the proof of the first item. Next, as discussed above Theorem 2.2 , the functions $\bar{A}(z)$ and $F(\bar{A}(z))$ are analytic on the disc $\left\{z \in \mathbb{C}:|z|<R_{G}\right\}$. Recalling that $\bar{A}$ is the inverse of $A$, we get that $\bar{A}(0)=\theta, \bar{A}^{\prime}(0)=A^{\prime}(0)^{-1}$ and $F(\theta)=0$. An application of the Taylor expansion to the right-hand side of (2.18) yields

$$
\mathcal{L}_{n}(x)=\frac{1}{n !}\left(\frac{\partial^{n}}{\partial z^{n}} e^{F(\bar{A}(z))-x(\bar{A}(z)-\theta)}\right)_{z=0} .
$$

Thus, recalling that $\mathcal{L}_{n}(x)=\sum_{k=0}^{n} a_{k, n} x^{k}$, we further see that $a_{n, n}=\frac{(-1)^{n}}{n !} e^{F(\bar{A}(0))}\left(\bar{A}^{\prime}(0)\right)^{n}$ so that (3.4) holds. Next we determine the remaining coefficients $a_{0, n}, \ldots, a_{n-1, n}$.

Using (2.2), (2.3), (2.4) and Leibniz's rule and with $\partial_{+}$denoting a (partial) right-derivative, and, $e_{k, \theta}(x)=x^{k} e^{-\theta x}, k \in \mathbb{N}$, we get

$$
\begin{aligned}
\mathcal{A L}_{n}^{\theta}(x) & =\sum_{k=0}^{n} a_{k, n} \lim _{t \downarrow 0} \frac{P_{t} e_{k, \theta}(x)-e_{k, \theta}(x)}{t}=\sum_{k=0}^{n} a_{k, n}(-1)^{k} \frac{\partial_{+}}{\partial t}\left(\frac{\partial_{+}^{k}}{\partial \lambda^{k}}\left(P_{t} e_{\lambda}(x)\right)_{\lambda=\theta}\right)_{t=0} \\
& =\sum_{k=0}^{n} a_{k, n}(-1)^{k} \frac{\partial_{+}^{k}}{\partial \lambda^{k}}\left(\frac{\partial_{+}}{\partial t}\left(P_{t} e_{\lambda}(x)\right)_{t=0}\right)_{\lambda=\theta} \\
& =\sum_{k=0}^{n} a_{k, n}(-1)^{k} \frac{\partial_{+}^{k}}{\partial \lambda^{k}}\left(\frac{\partial_{+}}{\partial t}\left(e^{-\Phi_{t}(\lambda)-\Psi_{t}(\lambda) x}\right)_{t=0}\right)_{\lambda=\theta}
\end{aligned}
$$

Copyright (C) by SIAM. Unauthorized reproduction of this article is prohibited. 


$$
\begin{aligned}
= & \sum_{k=0}^{n} a_{k, n}(-1)^{k} \frac{\partial_{+}^{k}}{\partial \lambda^{k}}\left(e^{-\lambda x}(\psi(\lambda) x-\phi(\lambda))\right)_{\lambda=\theta} \\
= & \sum_{k=0}^{n} a_{k, n}(-1)^{k} \sum_{j=0}^{k}\left(\begin{array}{c}
k \\
j
\end{array}\right)(-1)^{j} x^{j} e^{-\theta x}\left(x \psi^{(k-j)}(\theta)-\phi^{(k-j)}(\theta)\right) \\
= & e^{-\theta x} \sum_{j=0}^{n} \sum_{k=j}^{n}(-1)^{k-j} a_{k, n}\left(\begin{array}{c}
k \\
j
\end{array}\right) x^{j}\left(x \psi^{(k-j)}(\theta)-\phi^{(k-j)}(\theta)\right) \\
= & e^{-\theta x}\left(\sum_{j=1}^{n} x^{j}\left(\sum_{k=j}^{n}(-1)^{k+1-j} a_{k, n}\left(\left(\begin{array}{c}
k \\
j-1
\end{array}\right) \psi^{(k+1-j)}(\theta)+\left(\begin{array}{c}
k \\
j
\end{array}\right) \phi^{(k-j)}(\theta)\right)\right)\right. \\
& \left.+\left(\sum_{k=0}^{n}(-1)^{k+1} a_{k, n} \phi^{(k)}(\theta)\right)\right),
\end{aligned}
$$

where the interchanging of the derivative and expectation in the second equality follows by a standard argument as $\theta>0$, the switching of the two partial derivatives in the third equality is justified by the smoothness of the CBI exponents (see, e.g., Duffie, Filipović, and Schachermayer [8, Lemma 6.5(i)]) and for the last equality we used the identity $\psi(\theta)=0$. On the other hand, from (3.3), we have

$$
\mathcal{A L}_{n}^{\theta}(x)=-\lambda_{n} e^{-\theta x} \sum_{j=0}^{n} a_{j, n} x^{j}
$$

Comparing (A.5) and the last equality, we obtain (3.5). Note that $\mathbf{B}$ is invertible since it is upper-triangular with nonzero diagonal elements, which completes the proof of the proposition.

A.5. Proof of Proposition 3.2. We only prove the first identity since the second follows by similar arguments. Since $b>0$, we can pick $\rho>0$ big enough such that $(\rho+1) b>\lambda_{0}$. For such $\rho$, we have, with $|\mathcal{V}|$ denoting the total variation of the measure $\mathcal{V}$,

$$
\begin{aligned}
\int_{0}^{\infty} \int_{0}^{\infty}\left|e^{-(\rho+\mathrm{i} u) k}\left(e^{-(a+b y)}-e^{-k}\right)_{+}\right| d k|\mathcal{V}|(d y) & =\int_{0}^{\infty} \int_{a+b y}^{\infty} e^{-\rho k}\left(e^{-(a+b y)}-e^{-k}\right) d k|\mathcal{V}|(d y) \\
& =\int_{0}^{\infty}\left(\frac{e^{-(\rho+1)(a+b y)}}{\rho}-\frac{e^{-(\rho+1)(a+b y)}}{\rho+1}\right)|\mathcal{V}|(d y) \\
& <\infty .
\end{aligned}
$$

Hence we can use the Fubini theorem to deduce that for $\rho>0$ big enough such that $(\rho+1) b>$ $\lambda_{0}$,

$$
\int_{0}^{\infty} \int_{0}^{\infty} e^{-(\rho+\mathrm{i} u) k}\left(e^{-(a+b y)}-e^{-k}\right)_{+} \mathcal{V}(d y) d k=\frac{e^{-(1+\rho+\mathrm{i} u) a} \widehat{\mathcal{V}}(b(1+\rho+\mathrm{i} u))}{(\rho+\mathrm{i} u)(1+\rho+\mathrm{i} u)}
$$

Let $f(k)=e^{-\rho k} \int_{0}^{\infty}\left(e^{-(a+b y)}-e^{-k}\right)_{+} \mathcal{V}(d y)$ and let $\widehat{f}(u)=\frac{e^{-(1+\rho+\mathrm{i} u) a} \widehat{\mathcal{V}}(b(1+\rho+\mathrm{i} u))}{(\rho+\mathrm{i} u)(1+\rho+\mathrm{i} u)}$ be its Fourier transform. We have by the Jensen inequality and the Tonelli theorem for $\rho>0$ big enough such that $(\rho+1) b>\lambda_{0}$, 


$$
\begin{aligned}
\int_{-\infty}^{\infty}|\widehat{f}(u)| d u & \leq \int_{0}^{\infty} \int_{-\infty}^{\infty}\left|\frac{e^{-(1+\rho+\mathrm{i} u)(a+b y)}}{(\rho+\mathrm{i} u)(1+\rho+\mathrm{i} u)}\right| d u|\mathcal{V}|(d y) \\
& =\int_{0}^{\infty} \int_{-\infty}^{\infty} \frac{1}{\sqrt{\rho^{2}+u^{2}}} \frac{1}{\sqrt{(1+\rho)^{2}+u^{2}}} e^{-(\rho+1)(a+b y)} d u|\mathcal{V}|(d y) \\
& =e^{-(p+1) a} \int_{0}^{\infty} e^{-(\rho+1) b y}|\mathcal{V}|(d y) \int_{-\infty}^{\infty} \frac{1}{\sqrt{\rho^{2}+u^{2}}} \frac{1}{\sqrt{(1+\rho)^{2}+u^{2}}} d u<\infty
\end{aligned}
$$

Hence we can use the Fourier inversion theorem (cf. [13, Theorem 8.26]) to deduce for $k \in \mathbb{R}$ (noting that $f$ is continuous) that

$$
\int_{0}^{\infty}\left(e^{-(a+b y)}-e^{-k}\right)_{+} \mathcal{V}(d y)=e^{\rho k} f(k)=e^{\rho k} \frac{1}{2 \pi} \int_{-\infty}^{\infty} e^{\mathrm{i} u k} \widehat{f}(u) d u
$$

which together with the formula for $\widehat{f}(u)$ leads to the desired identity.

Acknowledgment. The authors would like to thank two anonymous referees for their valuable comments and suggestions on an earlier version that have led to an improvement in the quality of the paper.

\section{REFERENCES}

[1] R. M. Blumenthal and R. K. Getoor, Markov Processes and Potential Theory, Academic Press, New York, 1968.

[2] N. Boyarchenko And S. Levendorskil, The eigenfunction expansion method in multi-factor quadratic term structure models, Math. Finance, 17 (2007), pp. 503-539.

[3] P. CARR And D. B. MAdan, Option valuation using the Fast Fourier Transform, J. Comput. Finance, 2 (1999), pp. 61-73.

[4] M. Chazal, R. Loeffen, and P. Patie, Smoothness of continuous state branching with immigration semigroups, J. Math. Anal. Appl., 459 (2018), pp. 619-660.

[5] E. B. DAvies, Non-self-adjoint differential operators, Bull. London Math. Soc., 34 (2002), pp. 513-532.

[6] D. A. Dawson And Z. LI, Skew convolution semigroups and affine Markov processes, Ann. Probab., 34 (2006), pp. 1103-1142.

[7] D. Davydov And V. Linetsky, Pricing options on scalar diffusions: An eigenfunction expansion approach, Oper. Res., 51 (2003), pp. 185-209.

[8] D. Duffie, D. Filipović, and W. Schachermayer, Affine processes and applications in finance, Ann. Appl. Probab., 13 (2003), pp. 984-1053.

[9] D. Duffie And N. GÂRleanu, Risk and Valuation of Collateralized Debt Obligations, Financial Analysts J., 57, 2001, pp. 41-59.

[10] D. Duffie, J. PAn, And K. Singleton, Transform analysis and asset pricing for affine jump-diffusions, Econometrica, 68 (2000), pp. 1343-1376.

[11] D. Filipović, A general characterization of one factor affine term structure models, Finance Stoch., 5 (2001), pp. 389-412.

[12] L. N. G. FILON, On a quadrature formula for trigonometric integrals, Proc. Roy Soc. Edinburgh, 49 (1928), pp. 38-47.

[13] G. B. Folland, Real Analysis. Modern Techniques and Their Applications, 2nd ed., John Wiley \& Sons, New York, 1999.

[14] V. Gorovoi And V. Linetsky, Black's model of interest rates as options, eigenfunction expansions and Japanese interest rates, Math. Finance, 14 (2004), pp. 49-78.

[15] F. Jamshidian, An exact bond option formula, J. Finance, 44 (1989), pp. 205-209.

Copyright (c) by SIAM. Unauthorized reproduction of this article is prohibited. 
[16] R. A. Jarrow And S. M. Turnbull, Delta, Gamma and bucket hedging of interest rate derivatives, J. Appl. Math. Finance, 1, 1994, pp. 21-48.

[17] J. KALlSEN, A didactic note on affine stochastic volatility models, in From Stochastic Calculus to Mathematical Finance, Springer, New York, 2006, pp. 343-368.

[18] S. KARLin AND J. MCGREGOR, Spectral theory of branching processes. I. The case of discrete spectrum, Z. Wahrscheinlichkeitstheorie Verw. Gebiete, 5 (1966), pp. 6-33.

[19] K. KawaZu and S. Watanabe, Branching processes with immigration and related limit theorems, Theory Probab. Appl., 16 (1971), pp. 36-54.

[20] M. Keller-Ressel And E. Mayerhofer, Exponential moments of affine processes, Ann. Appl. Probab., 25 (2015), pp. 714-752.

[21] M. Keller-Ressel, A. Papapantoleon, and J. Teichmann, The affine LiBOR models, Math. Finance, 23 (2013), pp. 627-658.

[22] A. Kuznetsov, A. Kyprianou, and V. Rivero, The Theory of Scale Functions for Spectrally Negative Lévy Processes, in Levy Matters II, Lecture Notes in Math. 2061, Springer, New York, 2013, pp. $97-186$.

[23] A. Kyprianou, Introductory Lectures on Fluctuations of Lévy Process with Applications, Springer-Verlag, Berlin, 2006.

[24] R. W. LEE, Option pricing by transform methods: Extensions, unification, and error control, J. Comput. Finance, 7 (2004), pp. 51-86.

[25] Z.-H. LI, Branching processes with immigration and related topics, Front. Math. China, 1 (2006), pp. 73-97.

[26] V. Linetsky, Pricing equity derivatives subject to bankruptcy, Math. Finance, 16 (2006), pp. 255-282.

[27] H. P. McKeAn, JR., Elementary solutions for certain parabolic partial differential equations, Trans. Amer. Math. Soc., 82, 1956, pp. 519-548.

[28] Y. OgurA, Spectral representation for branching processes on the real half line, Publ. Res. Inst. Math. Sci., 5 (1969-1970), pp. 423-441.

[29] Y. OGurA, Spectral representation for branching processes with immigration on the real half line, Publ. Res. Inst. Math. Sci., 6 (1970), pp. 307-321.

[30] P. PATIE, Exponential functional of one-sided Lévy processes and self-similar continuous state branching processes with immigration, Bull. Sci. Math., 133 (2009), pp. 355-382.

[31] P. Patie And M. Savov, Spectral Expansion of Non-Self-Adjoint Generalized Laguerre SemiGroups, https://www.researchgate.net/publication/277712416_Spectral_expansions_of_non-self-adjoint_ generalized_Laguerre_semigroups (2015).

[32] P. Patie And M. SAvov, Cauchy problem of the non-self-adjoint Gauss-Laguerre semigroups and uniform bounds of generalized Laguerre polynomials, J. Spectr. Theory, 7 (2017), pp. 797-846.

[33] P. Patie And Y. Zhao, Spectral decomposition of fractional operators and a reflected stable semigroup, J. Differential Equations, 262 (2017), pp. 1690-1719.

[34] R: A Language and Environment for Statistical Computing, R Foundation for Statistical Computing, Vienna, Austria, 2015; available from https://www.R-project.org/.

[35] W. Rudin, Principles of Mathematical Analysis, 3rd ed., McGraw-Hill, New York, 1976.

[36] K. Soetaert, T. Petzoldt, and R. W. Setzer, Solving Differential Equations in R: Package deSolve, J. Statistical Software, 33 (2010), pp. 1-25.

Copyright (C) by SIAM. Unauthorized reproduction of this article is prohibited. 